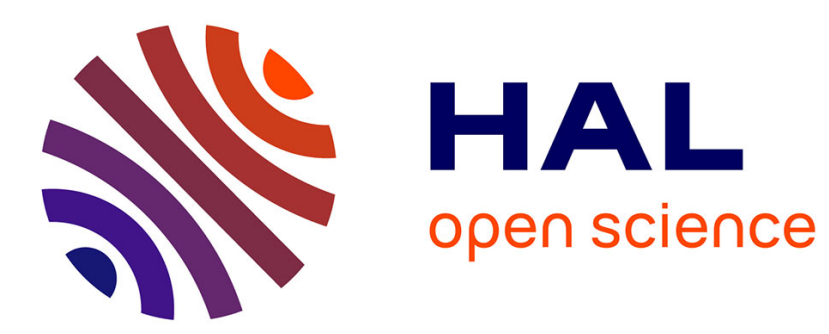

\title{
Coherent entropy induced and acoustic noise separation in compact nozzles
}

\author{
Wenjie Tao, Thierry Schuller, Maxime Huet, Franck Richecoeur
}

\section{To cite this version:}

Wenjie Tao, Thierry Schuller, Maxime Huet, Franck Richecoeur. Coherent entropy induced and acoustic noise separation in compact nozzles. Journal of Sound and Vibration, 2017, 10.1016/j.jsv.2017.01.012 . hal-01491836

\section{HAL Id: hal-01491836 \\ https://hal.science/hal-01491836}

Submitted on 29 Nov 2019

HAL is a multi-disciplinary open access archive for the deposit and dissemination of scientific research documents, whether they are published or not. The documents may come from teaching and research institutions in France or abroad, or from public or private research centers.
L'archive ouverte pluridisciplinaire HAL, est destinée au dépôt et à la diffusion de documents scientifiques de niveau recherche, publiés ou non, émanant des établissements d'enseignement et de recherche français ou étrangers, des laboratoires publics ou privés. 


\title{
Coherent entropy induced and acoustic noise separation in compact nozzles
}

\author{
Wenjie Tao ${ }^{a}$, Thierry Schuller ${ }^{\mathrm{a}}$, Maxime Huet ${ }^{\mathrm{b}}$, Franck Richecoeur ${ }^{\mathrm{a}, *}$ \\ a Laboratoire EM2C, CNRS, CentraleSupélec, Université Paris-Saclay, Grande Voie des Vignes, 92295 Châtenay-Malabry cedex, France \\ ${ }^{\mathrm{b}}$ ONERA - The French Aerospace Lab, F-92322 Châtillon, France
}

\begin{abstract}
A method to separate entropy induced noise from an acoustic pressure wave in an harmonically perturbed flow through a nozzle is presented. It is tested on an original experimental setup generating simultaneously acoustic and temperature fluctuations in an air flow that is accelerated by a convergent nozzle. The setup mimics the direct and indirect noise contributions to the acoustic pressure field in a confined combustion chamber by producing synchronized acoustic and temperature fluctuations, without dealing with the complexity of the combustion process. It allows generating temperature fluctuations with amplitude up to $10 \mathrm{~K}$ in the frequency range from 10 to $100 \mathrm{~Hz}$. The noise separation technique uses experiments with and without temperature fluctuations to determine the relative level of acoustic and entropy fluctuations in the system and to identify the nozzle response to these forcing waves. It requires multi-point measurements of acoustic pressure and temperature. The separation method is first validated with direct numerical simulations of the nonlinear Euler equations. These simulations are used to investigate the conditions for which the separation technique is valid and yield similar trends as the experiments for the investigated flow operating conditions. The separation method then gives successfully the acoustic reflection coefficient but does not recover the same entropy reflection coefficient as predicted by the compact nozzle theory due to the sensitivity of the method to signal noises in the explored experimental conditions. This methodology provides a framework for experimental investigation of direct and indirect combustion noises originating from synchronized perturbations.
\end{abstract}

\section{Introduction}

Turbulent flames give rise to fluctuations of pressure, temperature and velocity in the vicinity of the combustion zone. This generates acoustic, entropy and vorticity waves travelling at different speeds and directions in the combustion chamber. Acoustic waves propagate upstream and downstream the flame region, while entropy and vorticity waves are convected by the mean flow in the downstream direction. Coupling mechanisms between these waves are well known [1,2], and their interactions may contribute to increase noise emission or may dangerously increase resonant pressure oscillations in the combustion chamber [3]. For the noise generation process, one generally makes the distinction between direct combustion noise and indirect combustion noise.

\footnotetext{
* Corresponding author.

E-mail address: franck.richecoeur@centralesupelec.fr (F. Richecoeur).
} 
Direct combustion noise results from the unsteady heat release in the reaction zone [3-5]. Mechanisms at the origin of indirect combustion noise are more subtle. In aero-engines or ground based turbines, the flame and secondary air injection also produce entropy disturbances, which are convected through downstream flow-accelerating elements such as turbine blades or a converging nozzle. In these cases, a conversion of accelerated entropy waves into acoustic waves takes place, which has two possible consequences depending essentially on the outlet impedance of the combustor [1].

When the combustor exhaust reflection coefficient is small enough, indirect acoustic waves feature a relatively large spectral low frequency content and are essentially transmitted out of the chamber. This can be considered as an open-loop mechanism where entropy perturbations are converted into sound, which is then radiated in the surrounding environment. This contribution corresponds to the so-called indirect combustion noise. Different numerical strategies were developed to compute simultaneously the two types of noise in a combustion chamber and compare the relative sound pressure levels from both sources (see for example [6,7]). The corresponding experiments remain however a great challenge. To study indirect combustion noise alone, it is necessary to generate temperature fluctuations without any other acoustic source. Attempts have been made in early investigations [8] with clever setups generating entropy fluctuations by periodical heat addition to the air flow. The more recent Entropy Wave Generator (EWG) [9] uses an electrical heating module to produce a hot air column that is transported downstream through a convergent-divergent nozzle. The amplitude and spectral content of the entropy induced pressure wave propagating in the downstream direction through the nozzle was found in agreement with theoretical predictions. Numerical simulations of the EWG setup in supersonic cases [10] and subsonic cases [11,12] however also revealed that reflections from the EWG outlet and direct noise generated by the electrical heating system both contribute to pressure fluctuations in the discharge flow. It appears to be very difficult to generate pure indirect noise without introducing any additional acoustic disturbance. It is then worth examining experimental methods aiming at characterizing entropy noise when other noise contributions are present at the same time. This type of problem has only been addressed in a limited number of investigations, that generally rely on a separation of incoherent contributions to the noise field [13,14]. An alternative method is presented in this article based on the use of synchronized coherent harmonic pressure and entropy fluctuations. The validation of this method represents one of the motivations for the present study.

When the reflection coefficient of the combustor outlet increases, a coupling mechanism between the acoustic field and entropy fluctuations may also take place leading to amplified entropy disturbances at specific discrete tones. This feedback results often in a resonant loop, called rumble, where synchronized pressure and temperature oscillations increase in the chamber until a limit cycle is reached $[15,16]$. The role of entropy waves on the development of combustion instabilities has been investigated in many studies, but without providing definitive conclusions as will be emphasized below. Gaining insight into the coupling between acoustic and entropy waves during self-sustained thermoacoustic oscillations constitutes the second motivation for the development of a methodology and a setup allowing the separation of synchronized acoustic and entropy harmonic disturbances.

The following section makes a review of the state of knowledge on indirect combustion noise, i.e. entropy induced noise. In Section 3, the theoretical formalism is introduced together with definitions of the transmission and reflection coefficients used in this study. The experimental test bench is then presented in Section 4 together with a parametric analysis delimiting the domain of operation of this system. In Section 5, the separation technique is presented and validated with numerical simulations. Experimental results are presented in Section 6 and are discussed in Section 7 with the support of theoretical and simulation results.

\section{Entropy induced noise}

Since late 1960, efforts have been made to compute or measure impedances of nozzles and turbine blades. Tsien [17] first characterized analytically nozzle responses to one dimensional axial pressure and velocity perturbations by a transfer function defined as the ratio of mass flow perturbations to pressure perturbations at the entrance of the nozzle, or equally by the ratio $\left(\rho^{\prime}\left|\rho+u^{\prime}\right| u\right) /\left(p^{\prime} / p\right)$ where $\rho, u$ and $p$ are the density, velocity and pressure while the prime subscript denotes the corresponding fluctuating part, as a function of the oscillation frequency. Crocco and Cheng [18] later examined the specific admittance ratio $\alpha=\left(u^{\prime} / u\right) /\left(\rho^{\prime} \mid \rho\right)$ for studying the role of nozzle in combustion instabilities. They obtained an analytical solution for a subsonic nozzle in the entire frequency range under the hypothesis of isentropic small-amplitude perturbations and determined experimentally the admittance of a choked nozzle from pressure and velocity measurements made at the nozzle inlet. Bell et al. [19] examined experimentally the admittance of a nozzle mounted on a classical impedance tube facility. The standing-wave pattern that was superimposed on a mean flow velocity in the impedance tube was measured by a 10-microphone array to calculate the nozzle admittance using a linear regression method of the measured pressure amplitudes. Marble and Candel [1] simplified the problem by considering the nozzle as compact, with respect to the acoustic and entropy disturbance wavelengths. They considered incoming one-dimensional small perturbations of pressure and temperature, and compute analytically the reflection and transmission coefficients of a compact nozzle with jump conditions. Results show that, at the nozzle location, the incident acoustic wave is transmitted and reflected and an additional acoustic disturbance is generated due to the acceleration of entropy waves convected through the nozzle. In this compact approximation limit, the nozzle transfer function only depends on the nozzle inlet and outlet Mach numbers. In the same study, they also worked out on finite-length supercritical nozzles by assuming a one-dimensional linear velocity distribution in the nozzle [17]. Moase et al. [20] gave an analytical expression for the forced 
response of a choked nozzle and supersonic diffuser with a normal shock by considering the flow in the nozzle as multiple connected segments with linear evolution of the mean velocity. Stow et al. [21] showed that at moderate compactness ratio a simple choked nozzle can be represented by a constant cross section duct with an effective length that is terminated by the choked boundary condition. This effective length is found to be independent of the type of flow perturbation (acoustic or entropy) and can be calculated for any mean velocity profile in the nozzle. Goh and Morgans [22] later proposed to calculate the effective lengths for acoustic and entropy disturbances separately in supersonic nozzles. These developments were carried out in the linear acoustic regime with small-amplitude pressure, velocity and temperature disturbances. For waves with finite amplitudes propagating through a compact nozzle, Huet and Giauque [23], Durán et al. [11] recently suggested the use of a nozzle describing function in which acoustic waves are written in their Riemann invariant form. In this description, the extended nozzle describing function for entropy noise is a function of the entropy wave amplitude. Predictions with this formalism have been validated numerically in both subsonic and supersonic nozzles with finite amplitude waves.

Less progress were made on the experimental side due to the difficulty to separate the direct and indirect contributions to the sound field. Muthukrishnan et al. [13] carried out experiments on real engines to separate the entropy induced noise contribution from the total combustion noise inside a combustor. This was achieved by correlating the pressure signals recorded outside and inside the combustion chamber. Comparisons of the two noise spectra showed a transition of dominance from direct noise to entropy noise when the combustor outlet is choked. This method is however not valid at low frequencies where the noise level is the largest because in this frequency range acoustic and temperature fluctuations have a strong coherence. This coherence has been reported as an error factor in measuring the transfer function of a choked nozzle in a combustion chamber operating at stable condition [14]. This measurement is still a challenge that we aim at addressing here.

Dealing with entropy wave propagation and entropy noise contributions appears necessary for the examination of thermoacoustic instabilities in systems with sizeable flow acceleration of the burnt gases. In a generic combustor, Dowling [24] compared the thermoacoustic modes calculated with and without taking into account convective effects and found significant differences on the observed unstable modes when the flow Mach number increases. In the presence of a mean flow, the frequency of the first mode at a moderate temperature ratio across the heat release zone can be as two times smaller than in the no flow case. It was also found that this peak frequency is very sensitive to the heat release spatial distribution and little change occurred for a compact heat release zone much smaller than the acoustic and entropy wavelengths.

In aeronautical engines, the combustion chamber is optimized to be as short as possible and combustion takes place over a large volume of the chamber. This has motivated a series of studies within generic systems and the development of numerical tools to include the effects of entropy waves interacting with nozzles. Zhu et al. [15] observed a self-sustained low frequency $(50-120 \mathrm{~Hz})$ instability in a liquid-fueled combustor equipped with spray atomizers and chocked at its outlet. Analysis of the instability mechanism took into consideration the entropy mode and its convection time through the combustor was found to be close to the rumble instability period. Eckstein and Sattelmayer [16] compared the rumble instability frequency of a Rich-Quench-Lean (RQL) liquid-fueled combustor with a long exhaust open to the atmosphere and with a choked Venturi nozzle. Self-excited instabilities occurred in both cases and had comparable pressure amplitudes and frequencies, proving that the entropy mode was not the primary cause of the onset and sustainment of the rumble instability observed in this combustor. The different conclusions reached in [15] and [16] may be due to the dispersion of hotspots between the flame region where they are produced and the combustion chamber outlet due to turbulent mixing in the long RQL combustor. Dispersion and dissipation of entropy waves were also considered in the analysis of Goh and Morgans [25]. These authors found that the convected entropy mode can amplify or damp a thermoacoustic instability.

Motheau et al. [26] analyzed with a Helmholtz solver the effects of convection and reflection of entropy fluctuations on combustion instabilities within combustors featuring complex geometries. To this purpose, they defined a delayed entropy-coupled boundary condition (DECBC), which sums acoustic and entropy contributions to the reflected acoustic wave inside the chamber. These two contributions are linked to the incident velocity fluctuations upstream of the flame through different time-delayed models for acoustic and entropy waves and their respective reflection coefficients. This method naturally extends one dimensional analytical models to the simulation of these interactions within 3D geometries [27]. The DECBC then provides the acoustic-entropy interaction condition for their simulations with an Helmholtz solver in a zero-Mach number approach. Predicted unstable modes were found to be in good agreement with Large Eddy Simulations of the compressible reactive unsteady flow within the same model combustor. In their approach, the nozzle response to acoustic and entropy incident waves was shown to be essential in predicting successfully the correct combustion instability frequency.

In conclusion, entropy waves can transmit broadband noise outside the engine and there are evidences that they may also have an influence on the combustion chamber stability. Taking into account of the acoustic-entropy feedback appears necessary to predict combustion instabilities in chambers equipped with nozzles. The analytical and numerical models used for that purpose need as inputs a correct description of the transmission and reflection of entropy and acoustic waves at the combustion chamber outlet. The present work aims at developing an experimental framework to measure the acoustic and entropic impedances of a nozzle that is submitted to low frequency highly coherent pressure and temperature fluctuations. An original test bench generating synchronized harmonic pressure and temperature fluctuations has been developed to validate the methodology by comparison with the analytical results adapted from [1]. 


\section{Nozzle transfer functions}

The concept of transfer function is used in this work to separate direct and indirect contributions to the pressure field in the near field of a nozzle when it is submitted to harmonic acoustic and entropy disturbances simultaneously. A one dimensional approach is used. Pressure, velocity and temperature, are represented in this work as the sum of mean $\phi$ and time-varying $\phi^{\prime}$ components. The ratio $\phi^{\prime} \mid \phi$ is supposed to be limited to a few percents. Under these hypotheses, flow perturbations correspond to acoustic and entropy modes which propagate independently and are only coupled in the acceleration region within the nozzle [28]. Entropy fluctuations $s^{\prime}$ are convected by the mean flow at a velocity $u$ and acoustic fluctuations $p^{\prime}$ propagate with progressive and regressive components at the speed of sound $c$ with respect to the flow velocity $u$. The acoustic and entropy waves are first made dimensionless respectively by $p^{\prime} / \gamma p$ and $s^{\prime} / c_{p}$, where $\gamma$ and $c_{p}$ designate the specific heat ratio and the specific heat capacity at constant pressure of the fluid of interest. Their Fourier components are designated by $P$ and $\sigma$ at the angular frequency $\omega$ (with the convention $\exp (i \omega t)$ ).

A schematic with the different acoustic and entropy waves interacting with a nozzle is illustrated in Fig. 1. The nozzle is located at $x=x_{n}$ and is submitted to an incoming acoustic wave $P_{u}^{+}$and an entropy wave $\sigma$. In response, a reflected $P_{u}^{-}$and a transmitted $P_{d}^{+}$pressure waves are generated upstream and downstream the nozzle respectively. In the current work, the studied system includes a nozzle with a straight exhaust open to the atmosphere. There also exists a pressure wave $P_{d}^{-}$downstream of the nozzle that is generated by the reflective boundary condition $\left(p^{\prime}=0\right)$ at the system outlet. For a subsonic nozzle, this disturbance propagates towards the nozzle and can also generate a contribution to the sound field upstream the nozzle.

According to the noise generation mechanisms described in [1], the pressure waves $P_{u}^{-}$and $P_{d}^{+}$have direct and indirect contributions from the incident acoustic pressure and entropy waves. These pressure variables can be decomposed as:

$$
\begin{aligned}
& P_{u}^{-}=P_{u u}^{-}+P_{u d}^{-}+P_{u s}^{-} \\
& P_{d}^{+}=P_{d u}^{+}+P_{d d}^{+}+P_{d s}^{+}
\end{aligned}
$$

The first subscripts $u, d$ designate the regions upstream and downstream of the nozzle, the second subscripts $u, d, s$ stand for respectively the contributions to the sound field due to the incident acoustic wave from upstream, the incident acoustic wave from downstream and the entropy wave.

The expressions derived by Marble and Candel [1] for compact subsonic nozzles are used to separate the different contributions in the reflected $P_{u}^{-}$and transmitted acoustic waves $P_{d}^{+}$. As summarized in Eqs. (3)-(8), they only depend on the Mach numbers upstream and downstream of the nozzle $M_{u}$ and $M_{d}$ :

$$
\begin{aligned}
& R_{u}=\frac{P_{u u}^{-}}{P_{u}^{+}}=\left(\frac{M_{d}-M_{u}}{M_{d}+M_{u}}\right)\left(\frac{1+M_{u}}{1-M_{u}}\right)\left[\frac{1-\frac{1}{2}(\gamma-1) M_{u} M_{d}}{1+\frac{1}{2}(\gamma-1) M_{u} M_{d}}\right] \\
& T_{u}=\frac{P_{d u}^{+}}{P_{u}^{+}}=\left(\frac{2 M_{d}}{M_{d}+M_{u}}\right)\left(\frac{1+M_{u}}{1+M_{d}}\right)\left[\frac{1+\frac{1}{2}(\gamma-1) M_{d}^{2}}{1+\frac{1}{2}(\gamma-1) M_{u} M_{d}}\right] \\
& R_{d}=\frac{P_{d d}^{+}}{P_{d}^{-}}=-\left(\frac{M_{d}-M_{u}}{M_{d}+M_{u}}\right)\left(\frac{1-M_{d}}{1+M_{d}}\right)\left[\frac{1-\frac{1}{2}(\gamma-1) M_{u} M_{d}}{1+\frac{1}{2}(\gamma-1) M_{u} M_{d}}\right] \\
& T_{d}=\frac{P_{d u}^{-}}{P_{d}^{-}}=\left(\frac{2 M_{d}}{M_{d}+M_{u}}\right)\left(\frac{1-M_{d}}{1-M_{u}}\right)\left[\frac{1+\frac{1}{2}(\gamma-1) M_{u}^{2}}{1+\frac{1}{2}(\gamma-1) M_{u} M_{d}}\right]
\end{aligned}
$$

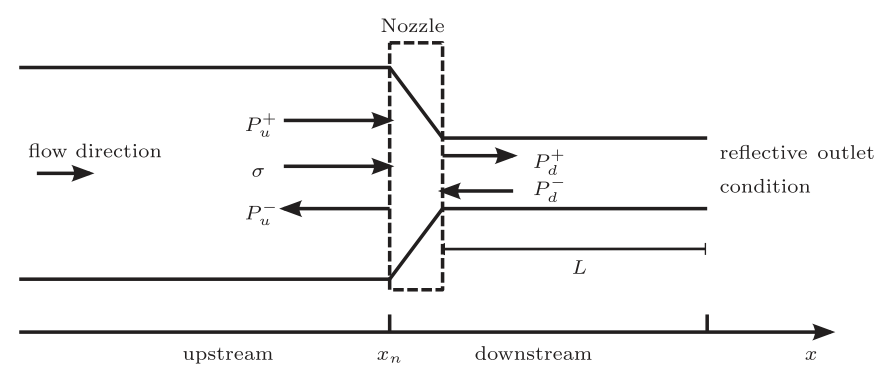

Fig. 1. Schematic view of waves in a nozzle. 


$$
\begin{aligned}
& R_{s}=\frac{P_{u s}^{-}}{\sigma}=-\left(\frac{M_{d}-M_{u}}{1-M_{u}}\right)\left[\frac{\frac{1}{2} M_{u}}{1+\frac{1}{2}(\gamma-1) M_{u} M_{d}}\right] \\
& T_{s}=\frac{P_{d s}^{+}}{\sigma}=\left(\frac{M_{d}-M_{u}}{1+M_{d}}\right)\left[\frac{\frac{1}{2} M_{d}}{1+\frac{1}{2}(\gamma-1) M_{u} M_{d}}\right]
\end{aligned}
$$

We consider now the outlet boundary condition downstream of the nozzle at $x=L$. For a nozzle that ends with a constant cross section area (see Fig. 1), the reflection coefficient $R_{L}$ on the boundary lying at a distance $L$ from the nozzle is given by:

$$
R_{L}=\frac{P_{d}^{-}}{P_{d}^{+}} \exp \left(i\left(k_{i}+k_{r}\right) L\right)
$$

where $k_{i}=\omega / c\left(1+M_{d}\right)$ denotes the wave number of the incident acoustic wave and $k_{r}=\omega / c\left(1-M_{d}\right)$ is the wave number of the reflected acoustic wave. At low frequency and for a low Mach number flow, $R_{L}$ can be set equal to -1 and $L$ needs to be corrected by an end correction $\delta$ [29,30]. Combining Eqs. (2), (7)-(9), the following linear system can be written:

$$
\left[\begin{array}{ccc}
1 & 0 & -T_{d} \\
0 & 1 & -R_{d} \\
0 & R_{L} e^{-i k_{i} L} & -e^{i k_{r} L}
\end{array}\right]\left[\begin{array}{c}
P_{u}^{-} \\
P_{d}^{+} \\
P_{d}^{-}
\end{array}\right]=\left[\begin{array}{cc}
R_{u} & R_{s} \\
T_{u} & T_{s} \\
0 & 0
\end{array}\right]\left[\begin{array}{c}
P_{u}^{+} \\
\sigma
\end{array}\right]
$$

An inversion of the square matrix on the left hand side of Eq. (10) yields the expressions of the reflected and transmitted acoustic waves $P_{u}^{-}$and $P_{d}^{+}$:

$$
\begin{aligned}
& P_{u}^{-}=\mathcal{R}_{a} P_{u}^{+}+\mathcal{R}_{s} \sigma \\
& P_{d}^{+}=\mathcal{T}_{a} P_{u}^{+}+\mathcal{T}_{s} \sigma
\end{aligned}
$$

where:

$$
\begin{aligned}
& \mathcal{R}_{a}=R_{u}-\frac{R_{L} T_{u} T_{d} e^{-i k_{i} L}}{R_{L} R_{d} e^{-i k_{i} L}-e^{i k_{r} L}} \\
& \mathcal{T}_{a}=\frac{T_{u} e^{i k_{L} L}}{R_{L} R_{d} e^{-i k_{i} L}-e^{i k_{r} L}} \\
& \mathcal{R}_{S}=R_{S}-\frac{R_{L} T_{s} T_{d} e^{-i k_{i} L}}{R_{L} R_{d} e^{-i k_{i} L}-e^{i k_{r} L}} \\
& \mathcal{T}_{s}=\frac{T_{s} e^{i k_{r} L}}{R_{L} R_{d} e^{-i k_{i} L}-e^{i k_{r} L}}
\end{aligned}
$$

The quantities $\mathcal{R}_{a}$ and $\mathcal{T}_{a}$ characterize the reflection and transmission coefficients of the incident acoustic waves from upstream of the nozzle. For convenience, $\mathcal{R}_{s}$ and $\mathcal{T}_{s}$ are called the indirect reflection and transmission coefficients and they characterize the acoustic waves generated by entropy fluctuations which propagate upstream and downstream of the nozzle. The ratio $\mathcal{R}_{a} / \mathcal{R}_{s}$ allows to compare the relative direct and indirect contributions to the boundary condition.

For a compact nozzle with a fixed geometry, Eqs. (3)-(8) combined with Eqs. (13)-(16) show that the four reflection and transmission coefficients only depend on the inlet and outlet Mach numbers of the flow. They are independent of how acoustic and entropy waves are generated.

In what follows a test bench has been developed allowing to generate harmonic acoustic and entropy waves in the flow approaching a nozzle. Entropy fluctuations are generated by hot jets in a colder crossflow where the main stream is pulsated with a loudspeaker. The separation of direct and indirect noise contributions in the upstream region of the nozzle is achieved by measuring the reflection coefficients $\mathcal{R}_{a}$ and $\mathcal{R}_{s}$ of the nozzle. The analytical expressions Eqs. (13) and (16) serve as references to validate the technique.

\section{TAFG: temperature and acoustic fluctuations generator}

The temperature and acoustic fluctuations generator (TAFG) is a system dedicated to simultaneously produce synchronized acoustic and entropy waves. As illustrated in Fig. 2(a), a loudspeaker fixed at the bottom of the TAFG is used to 
modulate the air flow $\dot{m}_{1}$ injected at ambient temperature $T_{1}$ in the lower part of the setup. The main stream crosses a honeycomb and a converging nozzle before entering a secondary injection zone where additional air is introduced by a series of jets in the main crossflow. This secondary air flow is created through an annular distribution unit made of thirty 1.5-mm holes shown in Fig. 2(b) and can be heated up to $473 \mathrm{~K}$. The acoustic modulation from the loudspeaker creates pressure fluctuations at the outlet of the secondary air injectors. Thus the jet flowrates are modulated at the same frequency as the acoustic wave propagating in the main stream. By elevating the temperature of the crossflow jets, temperature fluctuations in the main stream are created and are transported by the flow in the downstream direction. The perturbed

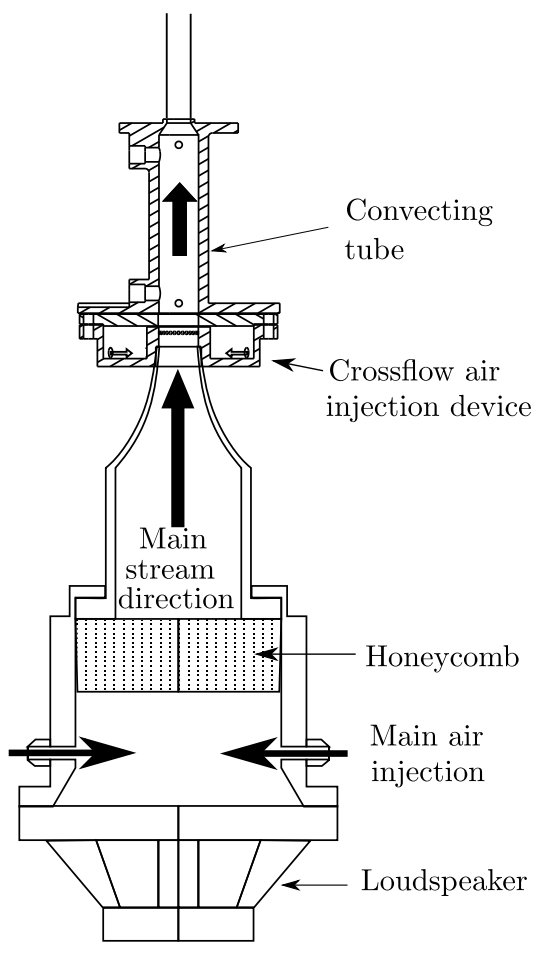

(a) Temperature and acoustic fluctuation generator (TAFG) creates coherent pressure and entropy waves accelerated by the final nozzle.

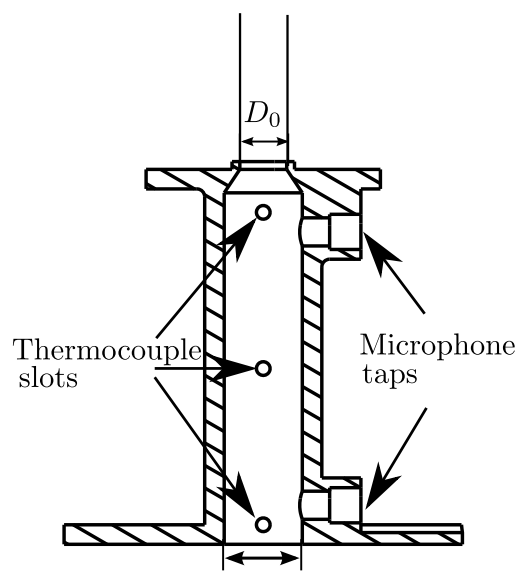

(c) The convecting tube ends with a converging nozzle to accelerate the flow

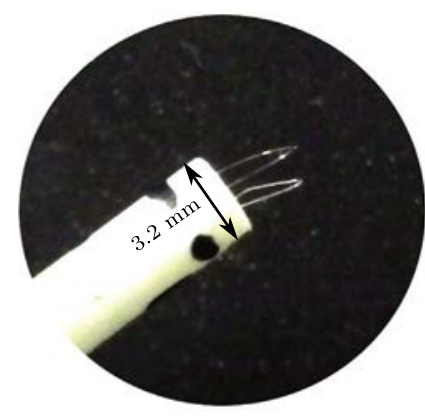

(d) To compensate the time delay of the temperature measurement, a twothermocouple sensor is used $(25 \mu \mathrm{m}$ and $50 \mu \mathrm{m})$.

Fig. 2. Components of the temperature and acoustic fluctuation generator (TAFG). 


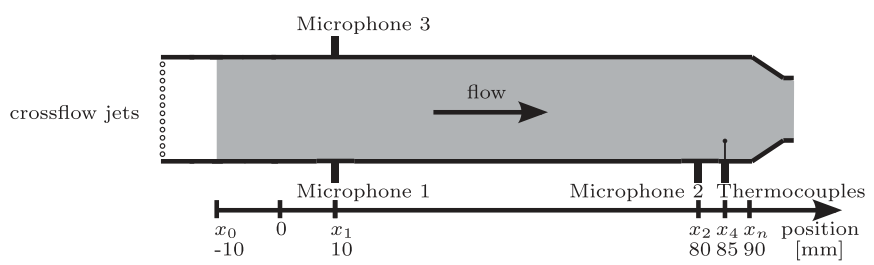

Fig. 3. Measurement positions presented in this work. The grey area corresponds to the numerical calculation domain for the SUNDAY code.

flow is conveyed through a $90 \mathrm{~mm}$ long tube of $20 \mathrm{~mm}$ diameter terminated by a convergent nozzle (see Fig. 2(c)) where entropy fluctuations are accelerated and converted to an indirect pressure wave. The nozzle ends with a tube extension of length $L=126.5 \mathrm{~mm}$ opened to atmosphere. Three different nozzles are tested. They all have the same length equal to $6 \mathrm{~mm}$, but feature different outlet diameters of $20 \mathrm{~mm}, 12 \mathrm{~mm}$ and $6 \mathrm{~mm}$. There is thus no acceleration with the $20 \mathrm{~mm}$ nozzle. These different terminations are designated respectively by D20, D12 and D06. The frequency range covered by the harmonic acoustic excitations goes from 10 to $100 \mathrm{~Hz}$. The loudspeaker is the direct acoustic source in the system, but it also generates synchronized temperature fluctuations. This configuration mimics highly coherent entropy and pressure waves as observed in some system featuring self-sustained combustion oscillations [31,32].

Sound pressure and temperature measurements are carried out to separate the direct and indirect contributions to the acoustic pressure by determining the direct $\mathcal{R}_{a}$ and indirect $\mathcal{R}_{s}$ nozzle reflection coefficients. Pressure taps and temperature slots are distributed along the 20-mm diameter tube shown in Fig. 2(c). The positions of the sensors are shown in Fig. 3. Pressure fluctuations are measured by two B\&K $1 / 4 \mathrm{in}$. microphones flush-mounted on the tube wall at positions $x_{1}$ and $x_{2}$. A third microphone of the same type is set at position $x_{1}$ in front of the first one as shown in Fig. 3. An interspace of $70 \mathrm{~mm}$ is chosen between the positions $x_{1}$ and $x_{2}$ to determine the acoustic reflection coefficient of the tube with the three-microphone technique described in [33]. Temperature is measured by two closely placed fast response K-type thermocouples (see Fig. 2(d)) with bead diameters of $25 \mu \mathrm{m}$ and $50 \mu \mathrm{m}$ at position $x_{4}$. The reason for employing two thermocouples is to compensate the effect of the delayed response of the thermocouples as will be discussed in Section 4.2. Measurements of temperature fluctuations at different radial positions in the cross section at $x_{4}$ were also carried out to check the uniformity of the temperature field and validate the planar wave hypothesis. The velocity in the tube is measured by a hot wire anemometer placed within the flow at $x_{4}$ at ambient air temperature. A set of dedicated experiments were performed to calibrate the setup and generate a constant velocity modulation level $u_{\text {rms }} / u$ independently of the forcing frequency.

Synchronized acoustic, velocity and temperature measurements are recorded at a sampling rate of $8192 \mathrm{~Hz}$ over a duration of 10 seconds. Two examples of results are shown in Fig. 4 for nozzles with different outlet diameters. The pressure signals feature clearly a sinusoidal shape at the modulation frequency. The temperature and velocity signals oscillate at the same fundamental frequency but the temperature signal features also a higher harmonic content and additional highfrequency perturbations that reduce the signal-to-noise ratio. A parametric study was carried out to optimize the setup in order to generate harmonic temperature oscillations with the highest amplitude.

\subsection{Parametric study of TAFG performance}

In order to generate temperature fluctuations as large as possible that can persist in the flow, several parameters have been varied to examine their influences on the resulting temperature fluctuations produced at the inlet of the nozzle. In these experiments, the forcing level $u_{r m s} / u_{\text {mean }}$ is kept constant and the crossflow air stream is heated up to $473 \mathrm{~K}$. The performance of the system is characterized by the root-mean-square (RMS) temperature fluctuations $T_{\mathrm{rms}}$ recorded at $x_{4}$ by the $25 \mu \mathrm{m}$ thermocouple.

Mixing of crossflow jets with a main stream has been extensively studied. The jet to main stream momentum flux ratio was found to be the main parameter controlling the jet penetration depth in the main stream. Experiments are first conducted with the D12 nozzle by varying the mass flow rates of the main stream $\dot{m}_{1}$ and of the crossflow jets $\dot{m}_{2}$, while the modulation frequency is fixed at $10 \mathrm{~Hz}$.

The RMS temperatures are measured for 25 couples of mass flow rates $\left(\dot{m}_{1}, \dot{m}_{2}\right)$. Fig. 5 shows that for each mass flow rate of the main stream there is an optimal mass flow rate for the crossflow jets yielding a maximum $T_{\text {rms }}$ fluctuation. The normalized amplitude of temperature fluctuations, defined by $T_{\mathrm{rms}} / T_{\text {mean }}$, is plotted in Fig. 6 as a function of the crossflow jets to main stream mass flow rate ratio $\dot{m}_{2} / \dot{m}_{1}$. This behavior is well predicted by a quasi-steady analysis of the TAFG test bench presented in Appendix A. Maximum fluctuations were found at $\dot{m}_{2} / \dot{m}_{1}=0.32$, although the values are largely scattered for the different total mass flow rates $\dot{m}_{1}+\dot{m}_{2}$ tested. It is suspected to be caused by a change of the turbulence level within the main stream that increases with the flow velocity. Turbulence smooths out any coherent stratifications of the temperature field produced by the modulated hot crossflow jets. These explorations show that the performance of the TAFG setup is to the leading order determined by the ratio of mass flow rates $\dot{m}_{2} / \dot{m}_{1}$ at $f=10 \mathrm{~Hz}$. This feature can be used to vary the total mass flow rate $\dot{m}_{1}+\dot{m}_{2}$ by maintaining the ratio $\dot{m}_{2} / \dot{m}_{1}$ constant. The ratio $\dot{m}_{2} / \dot{m}_{1}$ is fixed in the following experiments close to $\left(T_{1} / T_{2}\right)^{1 / 2}$ that maximizes temperature fluctuations $T_{\text {rms }}$ (See Appendix A).

These tests are repeated for different nozzle geometries and inlet Mach numbers $M_{u}$, and led to the choice of three 


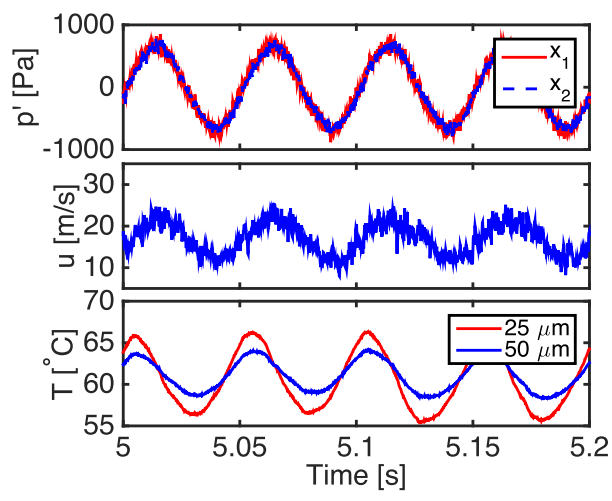

(a) Nozzle D12, $M_{u}=0.050, u_{\mathrm{rms}} / u=0.24$, modulation at $f=20 \mathrm{~Hz}$.

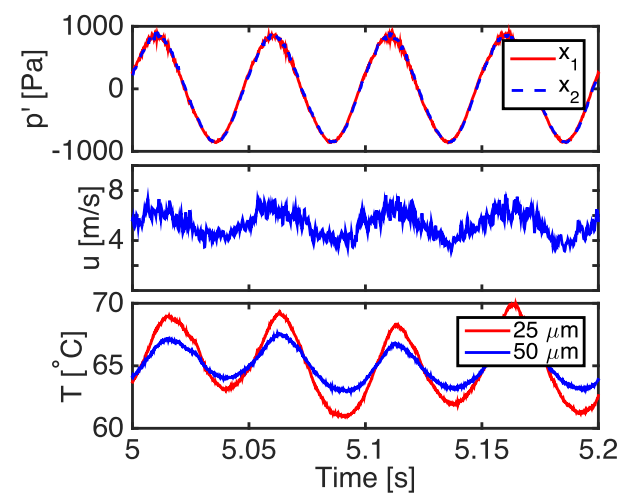

(b) Nozzle D06, $M_{u}=0.016, u_{\mathrm{rms}} / u=0.21$, modulation at $f=20 \mathrm{~Hz}$.

Fig. 4. Acoustic pressure, velocity and temperature measurements for two nozzle diameters.

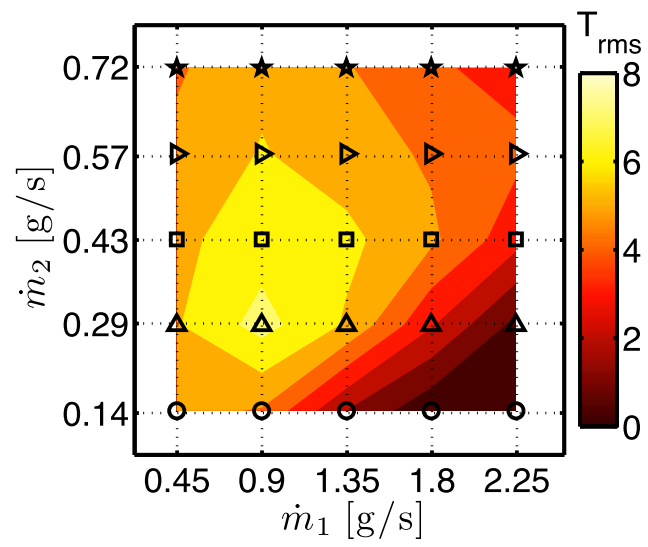

Fig. 5. Root-mean-square values of temperature fluctuations as a function of the mass flow rate of the main stream $\dot{m}_{1}$ and the crossflow jets $\dot{m}_{2} . f=10 \mathrm{~Hz}$. Nozzle D12.

optimal operating conditions summarized in Table 1 . The ratio $\dot{m}_{2} / \dot{m}_{1}$, is close to unity in the three cases.

For the three optimized operating conditions, Fig. 7 gives the RMS values of the temperature fluctuations reconstructed from the two thermocouple signals and the velocity modulation level $u_{\mathrm{rms}} / u$ as a function of the modulation frequency $f$. The modulation level of the velocity remains relatively constant at $20 \%$ over the whole frequency range. For frequencies above $40 \mathrm{~Hz}$ the system generates temperature fluctuations less than $8 \mathrm{~K}$. The hot crossflow jets only respond to low frequency pressure excitations. Modifying the hole diameter of the hot air injection unit or the number of holes did not change drastically the response of the system. This phenomenon narrows the frequency range that can be used to generate large amplitude temperature fluctuations. However for forcing frequencies around $10 \mathrm{~Hz}$, RMS temperature fluctuation levels larger than $8 \mathrm{~K}$ can easily be reached. This level is theoretically large enough to produce a noticeable contribution to indirect noise as shown by Bodony [34].

\subsection{Determination of upstream perturbation variables}

The synchronized pressure and temperature measurements are used in this section to determine the incident $P_{u}^{+}$and reflected $P_{u}^{-}$pressure waves and the entropy wave $\sigma$ at the forcing frequency $f$ in the region upstream of the nozzle.

\subsubsection{Measurement of the nozzle reflection coefficient}

The reflection coefficient $\mathcal{R}=P_{u}^{-} / P_{u}^{+}$at the nozzle inlet $x_{n}$ in Fig. 3 is first determined by the three-microphone method described in [33] with two microphones set at $x_{1}$ and one microphone set at $x_{2}$. Let $H_{21}$ be the complex ratio of pressure signals measured at position $x_{2}$ to that measured at position $x_{1}$. The reflection coefficient $\mathcal{R}$ is given by:

$$
\mathcal{R}=\frac{P_{u}^{-}}{P_{u}^{+}}=\frac{H_{21}-e^{-i k_{i} s}}{e^{i k_{r} s}-H_{21}} \cdot e^{-i\left(k_{i}+k_{r}\right)\left(x_{1}-x_{n}\right)}
$$




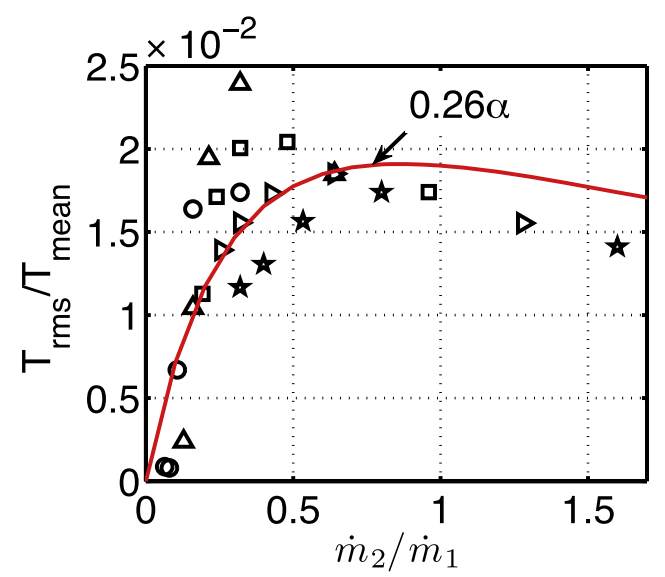

Fig. 6. Influence of the crossflow jet to main stream mass flow rate ratio $\dot{m}_{2} / \dot{m}_{1}$ on temperature fluctuations. $f=10 \mathrm{~Hz}$. Nozzle D12. The red curve is obtained by least-square fitting using $\alpha$ in Appendix A. (For interpretation of the references to color in this figure legend, the reader is referred to the web version of this article.)

Table 1

Optimized operation conditions of the TAFG setup.

\begin{tabular}{lllllllll}
\hline Case & $\begin{array}{l}\dot{m}_{1} \\
{[\mathrm{~g} / \mathrm{s}]}\end{array}$ & $\begin{array}{l}\dot{m}_{2} \\
{[\mathrm{~g} / \mathrm{s}]}\end{array}$ & $\dot{m}_{2} / \dot{m}_{1}$ & $\begin{array}{l}T_{\text {mean }} \\
{[\mathrm{K}]}\end{array}$ & $M_{u}^{\text {hot }}$ & $M_{u}^{\text {cold }}$ & $M_{u}$ & $M_{d}$ \\
\hline D12-1 & 0.81 & 0.89 & 1.1 & 342 & 0.018 & 0.017 & 0.012 \\
D12-2 & 2.69 & 2.69 & 1.0 & 333 & 0.053 & 0.050 & 0.038 \\
D06-1 & 0.81 & 0.89 & 1.1 & 339 & 0.017 & 0.016 & 0.012 & 0.106 \\
\hline
\end{tabular}

where $k_{i}=\omega / c\left(1+M_{u}\right), k_{r}=\omega / c\left(1-M_{u}\right)$ designate the wave numbers of the progressive and regressive acoustic waves in the upstream region of the nozzle, and $s$ is the inter-space of the two pressure sensors. To measure $H_{21}$ with accuracy, two sets of experiments are carried out. In the first set, measurements are conducted with the microphones set at their original location. The same measurements are then repeated by switching one microphone at location $x_{1}$ with the microphone set at location $x_{2}$. This switching method allows to compensate the phase distortion between the sensors [33]. The third microphone set at $x_{1}$ is used as a reference and allows to increase the signal to noise ratio [35].

\subsubsection{Estimation of the thermocouple response time}

The unsteady gas temperature $T_{w 1}$ and $T_{w 2}$, measured respectively by the $25 \mu \mathrm{m}$ and $50 \mu \mathrm{m}$ thermocouples are now used to estimate the response time of the thermocouples. The dynamic response of a thermocouple is well described [36-39] by:

$$
\frac{d T_{w}}{d t}=\frac{1}{\tau}\left(T_{g}-T_{w}\right)
$$

In this expression, $T_{g}$ stands for the gas temperature, $T_{w}$ is the thermocouple bead temperature and $\tau$ is the response time of the thermocouple. This response induces both amplitude and phase shift with respect to the gas temperature, which needs to be corrected. A Fourier transform of Eq. (18) yields the transfer function $H_{2 t c}=\hat{T}_{w 2} / \hat{T}_{w 1}$ between the thermocouples:

$$
H_{2 t c}=\frac{1+i \omega \tau_{1}}{1+i \omega \tau_{2}}=\frac{1+\omega^{2} \tau_{1} \tau_{2}}{1+\omega^{2} \tau_{2}^{2}}+i \frac{\omega\left(\tau_{1}-\tau_{2}\right)}{1+\omega^{2} \tau_{2}^{2}}=r+i x
$$

where the subscript 1 refers to the $25 \mu \mathrm{m}$ thermocouple and the subscript 2 to the $50 \mu \mathrm{m}$ thermocouple. It should be noted that the instrumental transfer functions of the thermocouple conditioners are considered here as the same. The real $(r)$ and imaginary $(x)$ components of $H_{2 t c}$ is then used to determine the time constants $\tau_{1}$ and $\tau_{2}$ as follows:

$$
\omega \tau_{2}=\frac{r-1}{x} \quad \omega \tau_{1}=\frac{r(r-1)}{x}+x
$$

Results for three cases studied in Table 1 are summarized in Table 2. They were deduced from the value of the transfer function $\mathrm{H}_{2 t c}$ at $10 \mathrm{~Hz}$ and supposed to be invariant with the modulation frequency. The true local gas temperature can then be recovered according to the Fourier transform of Eq. (18). A detailed examination of this method and its validation with other existing methods can be found in [40]. 


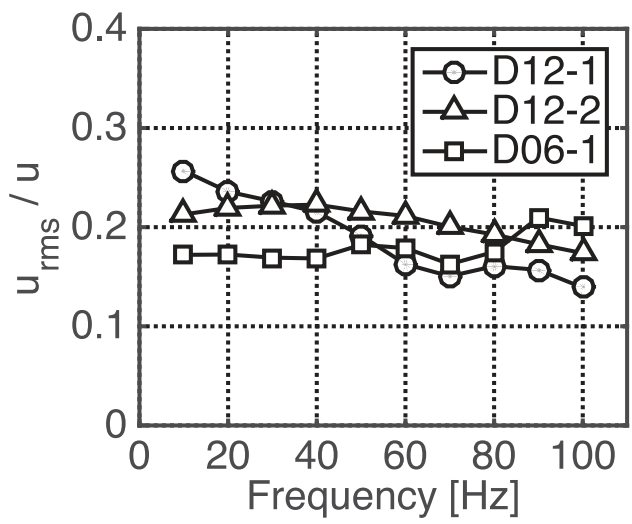

(a) Velocity modulation level

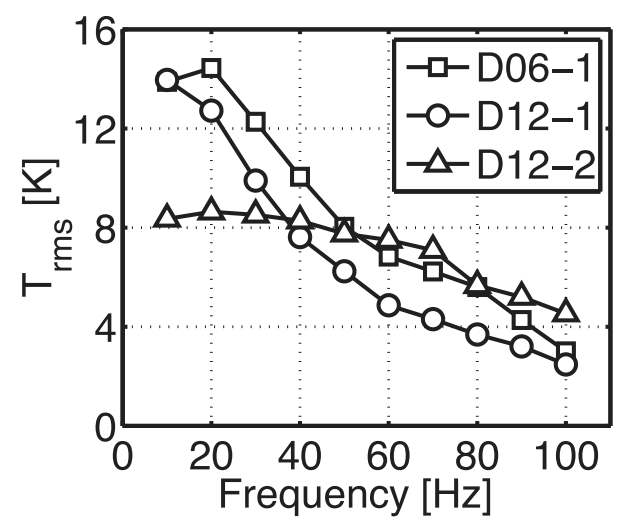

(b) Temperature fluctuations

Fig. 7. Velocity modulation level $u_{\mathrm{rms}} / u$ and root-mean-square compensated temperature fluctuation $T_{\mathrm{rms}}$ as a function of the forcing frequency for the optimized operating conditions.

\subsubsection{Determination of acoustic and entropy waves upstream of the nozzle}

It is now convenient to evaluate the entropy fluctuations from temperature and pressure fluctuations. Assuming smallamplitude perturbations in a perfect gas, one has:

$$
\frac{s^{\prime}}{c_{p}}=\frac{T^{\prime}}{T}-\frac{\gamma-1}{\gamma} \frac{p^{\prime}}{p}
$$

The Fourier transforms of the pressure and temperature signals thus write:

$$
\begin{aligned}
& \hat{p}_{1}=P_{u}^{+} e^{-i k_{i}\left(x_{1}-x_{n}\right)}+P_{u}^{-} e^{i k_{r}\left(x_{1}-x_{n}\right)} \hat{p}_{2} \\
& =P_{u}^{+} e^{-i k_{i}\left(x_{2}-x_{n}\right)}+P_{u}^{-} e^{i k_{r}\left(x_{2}-x_{n}\right)} \hat{T}_{g} \\
& =(\gamma-1)\left[P_{u}^{+} e^{-i k_{i}\left(x_{4}-x_{n}\right)}+P_{u}^{-} e^{i k_{r}\left(x_{4}-x_{n}\right)}\right]+\sigma e^{-i k_{s}\left(x_{4}-x_{n}\right)}
\end{aligned}
$$

where $k_{s}$ is the wave number of the entropy wave. Writing the complex ratio of the gas temperature and pressure signals at positions $x_{4}$ and $x_{2}$ respectively, one obtains:

$$
\frac{\hat{T}_{g}}{\hat{p}_{2}}=\frac{H_{s a} e^{-i k_{s}\left(x_{4}-x_{n}\right)}+(\gamma-1)\left(e^{-i k_{i}\left(x_{4}-x_{n}\right)}+\mathcal{R} e^{i k_{r}\left(x_{4}-x_{n}\right)}\right)}{e^{-i k_{i}\left(x_{2}-x_{n}\right)}+\mathcal{R} e^{i k_{r}\left(x_{2}-x_{n}\right)}}
$$

In this expression, the gas temperature $\hat{T}_{g}$ is unknown but can be estimated from the measured temperature signal $\hat{T}_{g}=\left(1+i \omega \tau_{1}\right) \hat{T}_{w 1}$ with the values in Table 2 . The nozzle reflection coefficient $\mathcal{R}$ evaluated at the inlet $x_{n}$ of the nozzle in Fig. 3 has been determined in Section 4.2.1. These quantities are used to deduce $H_{s a}=\sigma / P_{u}^{+}$, appearing in Eq. (22) and corresponding to the ratio of incident entropy to acoustic waves evaluated at $x_{n}$.

Fig. 8 gives the entropy wave to acoustic wave ratio for the operating conditions indicated in Table 1 . The modulus $\left|H_{s a}\right|$ and the phase angle $\varphi=\arg \left(H_{s a}\right)$ are plotted separately as a function of the forcing frequency. For the three configurations explored, the modulus of $H_{s a}$ decreases above $20 \mathrm{~Hz}$ as the modulation frequency rises. This low-pass filter behavior that was already illustrated in Section 4.1 is a characteristic of the TAFG system. High frequency temperature fluctuations are difficult to generate and are smoothed out due to mixing with the main stream, dispersion and dissipation. The case D121 yields the largest modulus $H_{s a}$ for all frequencies. Reducing the nozzle outlet diameter (D06-1) or raising the flow velocity (D12-2), leads to an increase of the mean pressure in the system. This causes a drop of $\Delta p$ and the amplitude of pressure fluctuations $p^{\prime}$ needs to be increased in order to maintain the same modulation level $u_{\text {rms }} / u$ (see Appendix A). The combination of these phenomena leads to the drop of the momentum of the cross flow jets, thereby the amplitude of the temperature fluctuations decreases in the TAFG system. The phase angle $\varphi$ varies quasi-linearly with the forcing frequency in Fig. 8 for the three cases explored and their phase velocities $\partial \varphi / \partial f$ are close to the phase change associated with

Table 2

Estimation of time constants using signals at $10 \mathrm{~Hz}$ modulation.

\begin{tabular}{llll}
\hline Cases & D12-1 & D12-2 & D06-1 \\
\hline$\tau_{1}[\mathrm{~ms}]$ & 45 & 18 & 44 \\
$\tau_{2}[\mathrm{~ms}]$ & 71 & 35 & 80 \\
\hline
\end{tabular}



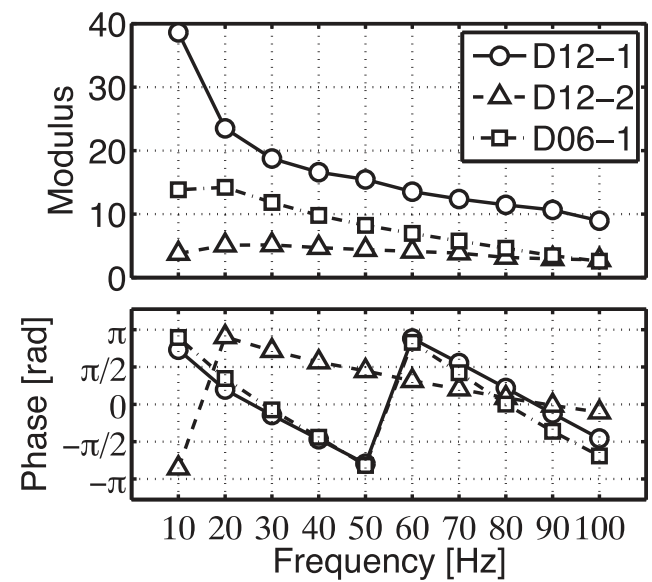

Fig. 8. Modulus and phase angle of the transfer function $H_{s a}=\sigma / P_{u}^{+}$.

the different propagation speeds of acoustic and entropy waves. The zero-frequency limit of the phase angle is $\pm \pi$ for the three operating conditions tested. This value corresponds well with the quasi-steady prediction of the TAFG setup presented in Appendix A.

The measurements of $\mathcal{R}$ and $H_{s a}$ are finally used to calculate the amplitude of the incident pressure wave $P_{u}^{+}$, the amplitude of the reflected pressure wave $P_{u}^{-}$and the entropy wave $\sigma$. The corresponding phase shifts $\varphi_{a}$ and $\varphi_{s}$ with respect to the incident acoustic wave at the nozzle inlet $x_{n}$ are also obtained for each modulation frequency ranging from $f=10$ to $100 \mathrm{~Hz}$. Results for $f=20$ and $f=100 \mathrm{~Hz}$ are listed in Table 3 together with the flow and forcing conditions.

These experiments allowed to determine the amplitudes of the incoming waves that can be generated by the TAFG. When interacting with the nozzle, they generate a reflected acoustic wave from which the entropic contribution needs to be extracted. The following section describes the separation method and uses the wave parameters as inputs of numerical simulations of the TAFG for validation.

\section{Separation method and numerical validation}

\subsection{Method for measuring direct and indirect nozzle reflection coefficients}

The reflection coefficient $\mathcal{R}$ at $x_{n}$ measured in the previous section is related to the complex ratio of incoming waves $H_{s a}$, the reflection coefficients $\mathcal{R}_{a}$ and $\mathcal{R}_{s}$ by:

$$
\mathcal{R}=\frac{P_{u u}^{-}+P_{u d}^{-}+P_{u s}^{-}}{P_{u}^{+}}=\frac{\mathcal{R}_{a} P_{u}^{+}+\mathcal{R}_{s} \sigma}{P_{u}^{+}}=\mathcal{R}_{a}+\mathcal{R}_{s} H_{s a}
$$

To determine the reflection coefficients $\mathcal{R}_{a}$ and $\mathcal{R}_{s}$, we compare results of two experiments carried out at a constant forcing level $u_{r m s} / u$, but with different entropy disturbances. Admitting linear perturbations, the direct and indirect contributions to the noise field add to each other. A natural choice is to conduct the first experiment with $\sigma=0$ (A) and a second one with $\sigma \neq 0$ (B). These two regimes can be obtained within the TAFG setup by maintaining the main stream and crossflow stream at either the same temperature (A) or different temperatures (B). In the first regime, without entropy perturbation $(\sigma=0)$, the reflection coefficient is given by:

$$
\mathcal{R}(\sigma=0)=\mathcal{R}_{a}
$$

For compact nozzles, it has been shown in Section 3 that the coefficients $\mathcal{R}_{a}$ and $\mathcal{R}_{s}$ only depend on the inlet and outlet Mach numbers of the nozzle considered and the boundary conditions at the nozzle outlet. This property allows to deduce the coefficient $\mathcal{R}_{s}$ from Eq. (24) using measurements in a second regime (B) with entropy disturbances. To do so, the mean flow in regimes (A) and (B) need to be the same, while the crossflow jets are injected at a different temperature.

Modifying the temperature of the crossflow jets alters the speed of sound in the flow upstream the nozzle. We estimated that the relative change of $\mathcal{R}_{a}$ between the regimes (A) and (B) varies between $2 \%$ and $10 \%$ due to to slight changes of the inlet Mach number $M_{u}$. Due to difficulties in controlling the temperature of the crossflow jets with a high precision, it was not possible to compensate this effect in the experiments carried out in this work. This phenomenon is thus treated here as a measurement uncertainty.

Finally by subtracting Eq. (24) from Eq. (23), one deduces: 
Table 3

Upstream perturbation variables at the inlet of the nozzle as a function of the flow and modulation conditions.

\begin{tabular}{|c|c|c|c|c|c|c|}
\hline Case & Config. & $u_{\mathrm{rms}} / u$ & $f[\mathrm{~Hz}]$ & $P_{u}^{+}$ & $P_{u}^{-} e^{i \varphi_{a}}$ & $\sigma e^{i \varphi_{S}}$ \\
\hline 1 & D12-1 & 0.24 & 20 & 0.0019 & $0.0014 e^{+3.12 i}$ & $0.0441 e^{+0.62 i}$ \\
\hline 2 & D12-1 & 0.14 & 100 & 0.0010 & $0.0008 e^{+3.06 i}$ & $0.0089 e^{-1.44 i}$ \\
\hline 3 & D12-2 & 0.22 & 20 & 0.0074 & $0.0040 e^{+2.57 i}$ & $0.0375 e^{+2.83 i}$ \\
\hline 4 & D12-2 & 0.17 & 100 & 0.0067 & $0.0028 e^{+2.89 i}$ & $0.0185 e^{-0.33 i}$ \\
\hline 5 & D06-1 & 0.17 & 20 & 0.0035 & $0.0021 e^{+0.93 i}$ & $0.0496 e^{+1.08 i}$ \\
\hline 6 & D06-1 & 0.20 & 100 & 0.0039 & $0.0015 e^{+0.37 i}$ & $0.0102 e^{-2.17 i}$ \\
\hline
\end{tabular}

$$
\mathcal{R}_{s}=\frac{\mathcal{R}(\sigma \neq 0)-\mathcal{R}(\sigma=0)}{H_{s a}}
$$

This method designated as diff- $R$ requires to measure the reflection coefficient for two different flow conditions together with the ratio of incoming waves. A numerical validation of the method is presented next.

\subsection{Numerical validation}

In this section, the method described above to measure nozzle reflection coefficients $\mathcal{R}_{a}$ and $\mathcal{R}_{s}$, is used to examine the numerical signals given by a dedicated code. The obtained nozzle reflection coefficients are compared with the analytical solutions.

The numerical solver used in this work is called SUNDAY (SimUlation of Noise in 1D flows due to fluctuAtions in entropY) $[12,23]$. It solves the quasi-one-dimensional nonlinear Euler equations governing the flow in channels with variable section area. It is capable of calculating the pressure and temperature fluctuations within the numerical domain for different nozzle geometries and flow operating conditions. It also allows to force the flow by acoustic and entropy waves with reflective or non-reflective boundary conditions at the extremities of the simulation domain. The boundary conditions used in this work are non-reflective at the entrance of the simulation domain and fully reflective at the exit (see Fig. 3). The investigated numerical domain corresponds to the part of TAFG setup downstream the hot air injection unit starting at $x_{0}$ (see Fig. 3 ), where it is assumed that flow disturbances are uniform and have a plane wave structure.

The numerical case shares the same mean flow condition as case 3 in Table 3 but with different wave amplitudes: $f=20 \mathrm{~Hz}, P_{u}^{+}=0.003, \sigma=-0.1, M_{u}=0.050, L+\delta=5.66 \mathrm{~mm}$ and $T_{\text {mean }}=473 \mathrm{~K}$. This configuration could not be explored experimentally and corresponds to an ideal operating condition, where the amplitude ratio $\eta$ of indirect to direct noise contribution reaches about $40 \%$ :

$$
\eta=\left|\frac{P_{u s}^{-}}{P_{u u}^{-}}\right|=\left|\frac{\mathcal{R}_{s}}{\mathcal{R}_{a}}\right| \cdot\left|\frac{\sigma}{P_{u}^{+}}\right|=\left|\frac{\mathcal{R}_{s}}{\mathcal{R}_{a}}\right| \cdot\left|H_{s a}\right| \simeq 0.40
$$

The RMS temperature fluctuations generated in this case reaches about $47 \mathrm{~K}$. Simulations are carried out first by only considering acoustic waves (A) and then with coexisting acoustic and entropy excitations (B). The numerical time series of pressure, velocity and temperature are recorded at the same positions as in the TAFG setup after the transient waves have been evacuated. These numerical signals are then sampled at $8192 \mathrm{~Hz}$ during $1 \mathrm{~s}$.

In order to mimic the experimental conditions, two levels of random noise are added to the ideal numerical signals. The ideal and noisy signals are then post-processed by a power spectral analysis with the Welch method. Results for the direct and indirect nozzle reflection coefficients are summarized in Fig. 9. The noise level is indicated in this figure by the signalto-noise ratio SNR in $\mathrm{dB}$. With ideal noise-free signals $(\mathrm{SNR}=\infty)$, the reflection coefficient $\mathcal{R}(\sigma=0)$ calculated in the simulation (A) is found to be equal to the analytical prediction of the acoustic reflection coefficient $\mathcal{R}_{a}$ from Eq. (13). The indirect reflection coefficient $\mathcal{R}_{s}$ calculated from simulations (B) also agrees well with its analytical prediction with a relative difference less than $4 \%$. The slightly overestimated value for $\mathcal{R}_{s}$ results from the difference between the nozzle outlet boundary condition implemented in SUNDAY code and the radiative impedance used the analytical solution (see Eq. (9)). Besides, the introduction of an entropy wave in simulation (B) largely modifies the nozzle acoustic response. A comparison between $\mathcal{R}(\sigma=0)$ and $\mathcal{R}(\sigma \neq 0)$ is shown in Fig. 9 (left) for the noise free signals. The magnitude of the reflection coefficient $\mathcal{R}$ deduced from the simulations with (B) entropy disturbances is much lower than that found without (A) entropy perturbations. When noise is added to the ideal excitation signals, the precision and accuracy on the modulus of $\mathcal{R}(\sigma=0), \mathcal{R}(\sigma \neq 0)$ and $\mathcal{R}_{S}$ are still in good agreement with the values found for the ideal signals in Fig. 9 with relative differences limited to a few percent. One thousand tests are performed with different white noises and the dispersion of the results is shown. Noise addition has a limited impact on the modulus of the reflection coefficient $\mathcal{R}$. Dispersion of the data is more important for the entropy modulus $\left|\mathcal{R}_{s}\right|$ even if the averaged value is consistent with the analytical prediction. The phase angle calculations are 


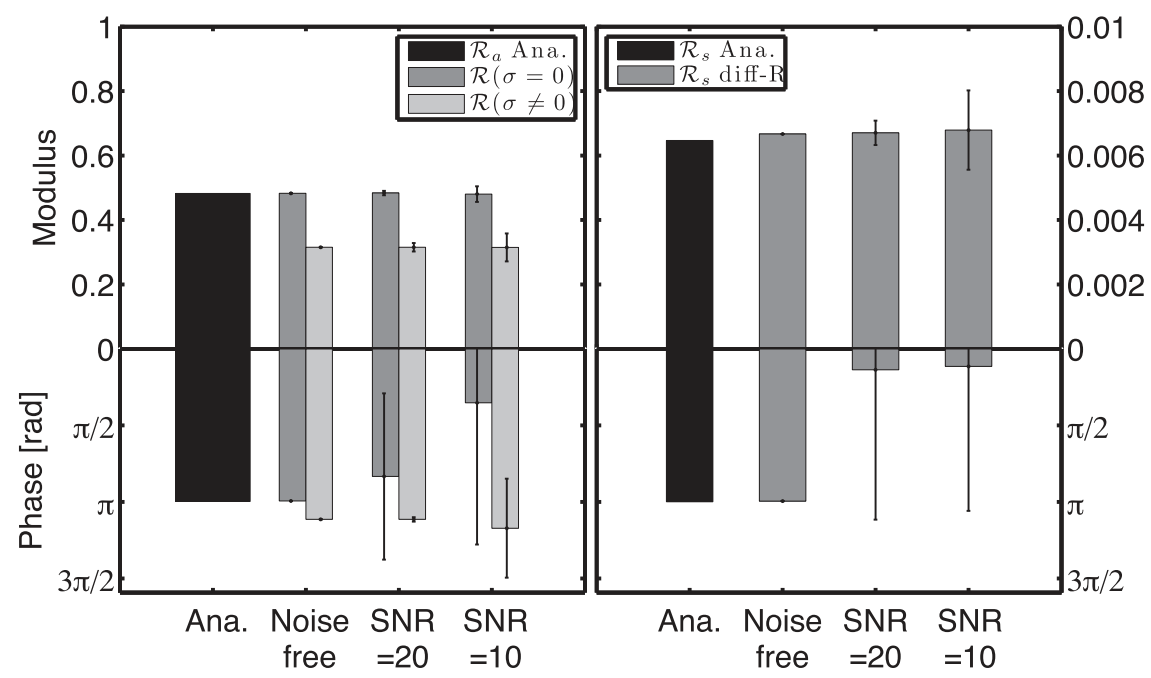

Fig. 9. Direct (left) and the indirect (right) reflection coefficients obtained from SUNDAY simulations with the diff-R method. Results are compared to analytical values of $\mathcal{R}_{a}$ and $\mathcal{R}_{s}$. Error bars are obtained from 1000 runs with random noise.

progressively altered for the two coefficients $\mathcal{R}$ and $\mathcal{R}_{s}$ when the SNR is reduced.

The diff- $R$ method combines pressure and temperature measurements with power spectral data processing to evaluate the acoustic and entropy reflection coefficients. The method has been validated by numerical simulations with ideal incoming waves of large amplitude. However, the measurement accuracy decreases when the signal quality worsen and the SNR falls below $20 \mathrm{~dB}$. The phase is more sensitive than the modulus. This method is tested on the TAFG setup in the next section.

\section{Experimental results}

Experimental signals are now analyzed for three operating conditions with different nozzles shown in Fig. 7.

The reflection coefficients at the nozzle inlet when the entropy mode is switched on $\mathcal{R}(\sigma \neq 0)$ and off $\mathcal{R}(\sigma=0)$ are presented first. Their modulus and phase angles are plotted in Fig. 10 together with the analytical values of $\mathcal{R}_{a}$. When the main stream and crossflow jets are at the same temperature, the measured reflection coefficient $\mathcal{R}(\sigma=0)$ corresponds well to the analytical value of $\mathcal{R}_{a}$ except at the very low frequency of $10 \mathrm{~Hz}$. It is known that the three-microphone technique has a limited spectral validity, especially at low frequency. Due to the limited distance $s=70 \mathrm{~mm}$ between the microphones 1 and 2 in Fig. 3, the relative phase variation between the two signals decreases at low frequency together with the measurement accuracy. The limited length of the setup does not allow to increase this distance. When air in the crossflow jets is injected at higher temperature, here fixed to $473 \mathrm{~K}$, a difference between the reflection coefficients $\mathcal{R}(\sigma \neq 0)$ and $\mathcal{R}(\sigma=0)$ can be observed. As expected, the difference is the largest at low frequency. It has been shown in the Section 4.2 that the amplitude of temperature fluctuations reaches a maximum at low frequency leading to high values of $\left|H_{s a}\right|$. The relative contribution of entropy to the generated noise is maximum at low modulation frequencies. We also notice that the entropic contribution is maximum with the 6-mm diameter nozzle (D06-1), the configuration for which the flow features the highest acceleration.

Eq. (25) is now used to deduce the entropic reflection coefficient $\mathcal{R}_{s}$ plotted in Fig. 11. Measurements largely differ from analytical predictions. Eq. (15) yields a modulus $\mathcal{R}_{S}=8 \cdot 5 \cdot 10^{-4}$ for the D12- 1 case and around $5 \cdot 10^{-3}$ for the D12-2 and D061 cases. These contributions are very small. The experimental results feature a large scatter. The modulus is overestimated by an order of magnitude. The modulus of the D12-2 and D06-1 cases however takes the same values in the measurements, and the modulus of the D12-1 case is smaller so that the hierarchy is the same in the experiments and predictions. To investigate these differences, two hypotheses have been tested with numerical simulations: (1) pressure and temperature measurements are too noisy to provide quantitative information and (2) non linear interactions take place between pressure, entropy and velocity disturbances.

\section{Discussion}

The SUNDAY code computes the acoustic and entropy propagation in a cylindrical chamber ended by a converging nozzle. Six different simulations are completed with the conditions listed in Table 3 to interpret the experimental results. The amplitude of the incoming harmonic acoustic $P_{u}^{+}$and entropy $\sigma$ waves are set in the simulations equal to the values 

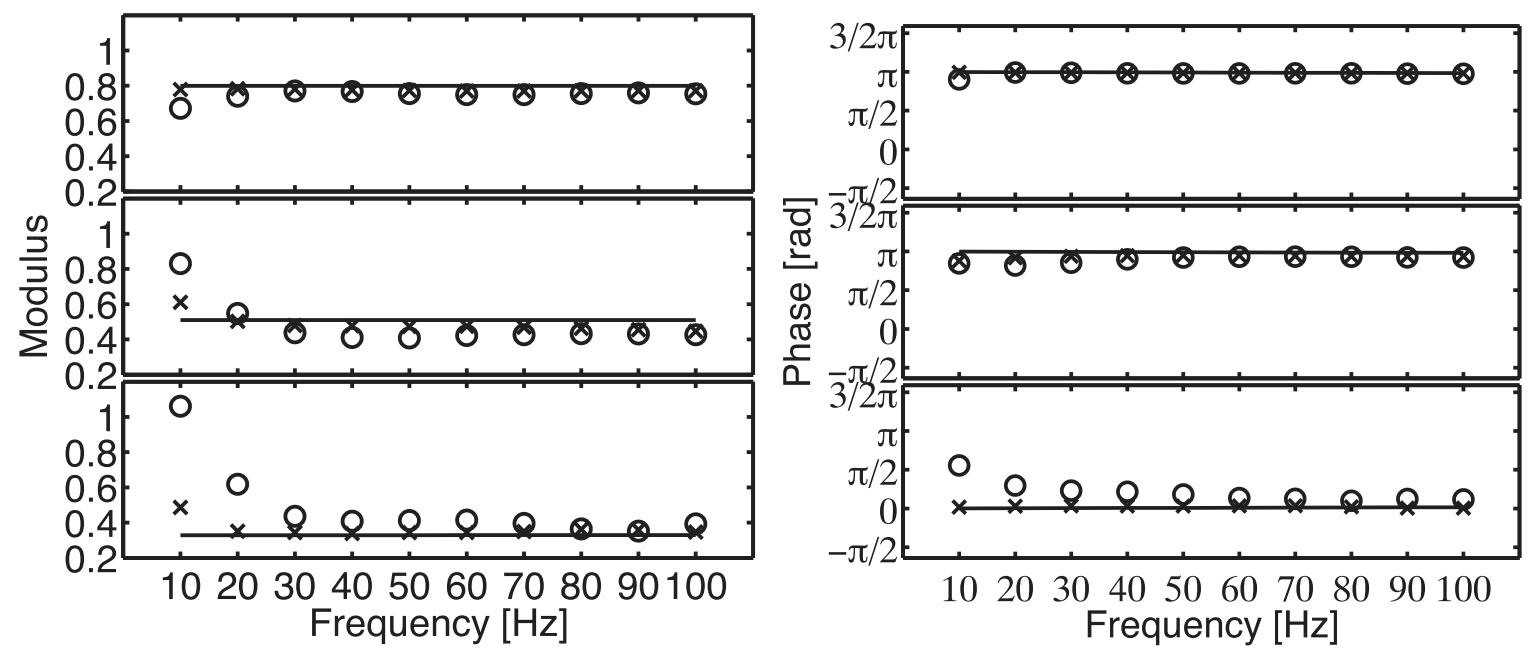

Fig. 10. Modulus (left) and phase angle (right) of the reflection coefficient for configurations D12-1 (top), D12-2 (middle) and D06-1 (bottom). Analytical predictions of $\mathcal{R}_{a}$ (blackline), $\mathcal{R}(\sigma \neq 0)(0)$ and $\mathcal{R}(\sigma=0)(\times)$.

deduced from experiments. The phase shift between acoustic and entropy waves at the inlet of the simulation domain located at $x_{0}$ is deduced from the value indicated at $x_{n}$ in Table 3 . The link between these two phase shifts is calculated by considering the propagation time lag between the acoustic wave travelling at the speed $u+c$ and the entropy wave convected at the bulk flow velocity $u$.

Numerical and experimental pressure, velocity and temperature time series are plotted in Fig. 12 for the case 1 in Table 3. These signals were first synchronized with the pressure measured at $x_{1}$. A good agreement between the ideal numerical and experimental signals is found. The phases between the different quantities and the amplitudes match well. This test shows that measurements capture the correct physics.

The noticeable difference between time series is the noise contained in experimental data and the wave steepening in the temperature signal. It is worth examining the signal-to-noise ratio (SNR) in the experiments. It has been shown in Section 5.2 that incoming waves with a low SNR strongly alter the numerical results when it drops below $20 \mathrm{~dB}$. Here, the SNR of measured pressure, velocity, temperature signals before applying the diff-R method reaches a maximum level of $20 \mathrm{~dB}$. To mimic this, a Gaussian noise with a level of $20 \mathrm{~dB}$ is added to numerical signals before applying the diff-R method. Results for the reflection coefficients deduced from ideal and noisy numerical signals are synthesized in Figs. 13 and 14. In Fig. 13, the reflection coefficient is shown for the six different conditions. The black bar corresponds to the analytical reference. The diff- $\mathrm{R}$ method applied with noise free signals match well the analytical values. Noise addition (white bars) affects the dispersion of the phase and modulus but the average values remain consistent with noise free signals. The entropy reflection coefficient is presented in Fig. 14 with the same color scale: the black bars are the analytical references, the gray bars denote the diff- $\mathrm{R}$ method with noise free signals and the white ones show the results obtained with the diff- $\mathrm{R}$ method and the noisy time series. The noise free simulations match well with the analytical results, a tiny phase difference is observed for the first two cases. This confirms the results obtained in Section 5.2 where an ideal entropy wave with an amplitude equal to $\sigma=0.1$ was used. In the present situation, the analysis is carried out for amplitudes of the entropy waves that were measured. It is thus worth noting that the diff-R method allows to catch small entropy contributions provided that the quality of the signal is good.

With a SNR equals to $20 \mathrm{~dB}$, the entropy coefficient modulus and phase are drastically changed in Fig. 14. We see again that when the SNR decreases the results are strongly altered. We can draw two conclusions about the experiment and signal quality: (1) The diff-R method is based on correlations between measurements of pressure and temperature signals. With noisy signals, the correlation varies significantly and this in turn modifies the coefficient values. To counter balance this, it is necessary either to create temperature fluctuations of higher amplitudes or to increase the number of sensors to increase the number of correlations and diminish noise effects. The small dimensions of the setup did not allow such modification. (2) In the present experiment, the acoustic pressure resulting from entropy acceleration is very low. By comparing the theoretical direct and indirect contributions to the reflected pressure wave, Eq. (26) indicates that the contribution from the indirect noise generated by entropy disturbances remains always lower than $25 \%$ of the pressure signal for all the six cases explored in Table 3. This setup creates small indirect noise sources but they remain too small to be accurately separated by the diff-R method. However, numerical simulations let us expect much better results when applied to a combustion chamber equipped with a large number of sensors and experiencing a larger entropy contribution to the produced noise.

Numerical simulations also point an interesting phenomenon that needs to be considered. While pressure and 

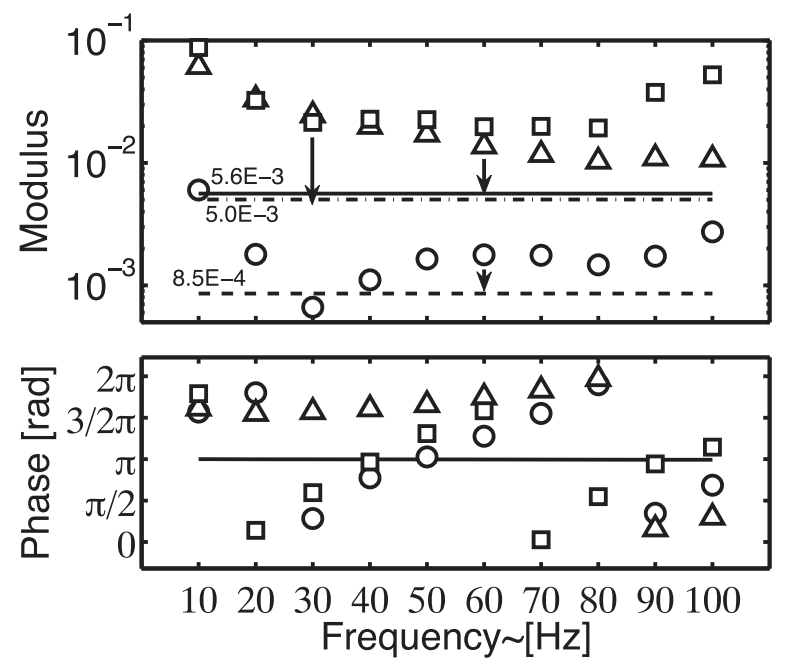

Fig. 11. Indirect reflection coefficient $\mathcal{R}_{s}$. D12-1 case: prediction (blackline), experiment (O). D12-2 case: prediction (dashed-line), experiment $(\Delta)$. D06-1 case: prediction (dash-dotted-line, experiment (ㅁ)).

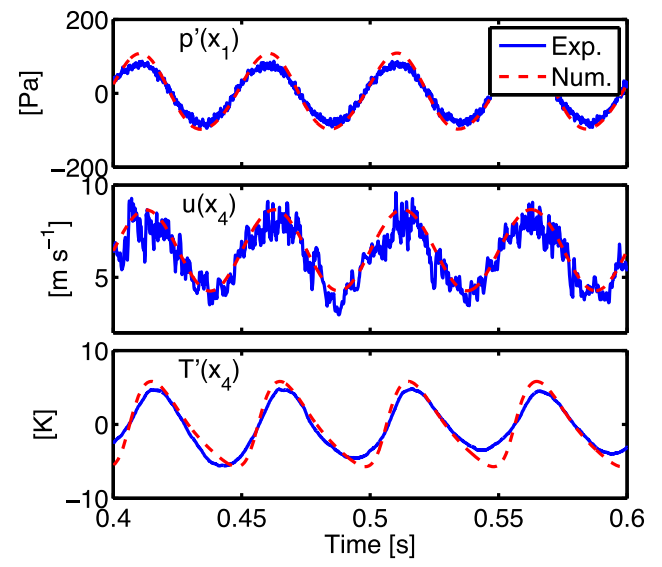

Fig. 12. Comparison between measurements and simulations for the case 1 in Table 3.

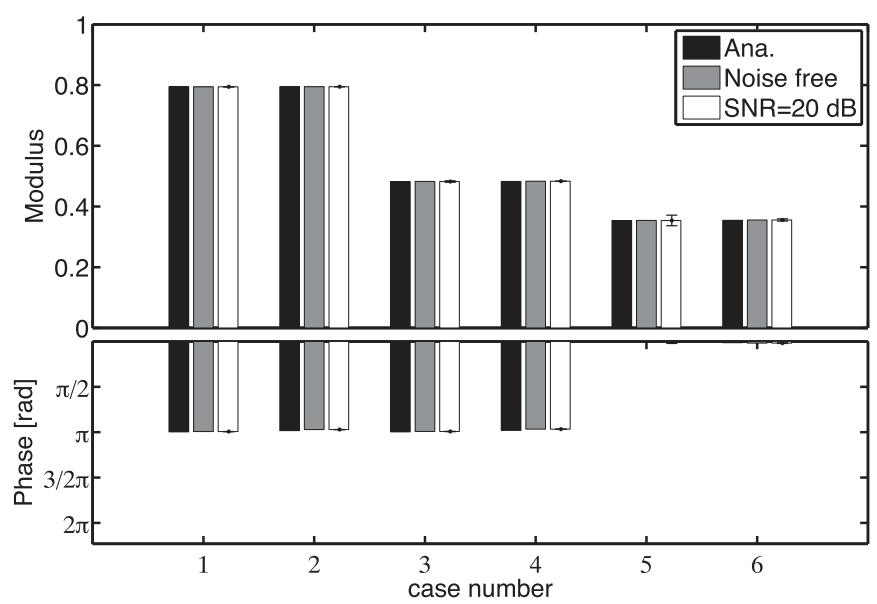

Fig. 13. Modulus (top) and phase (bottom) of the direct reflection coefficient $\mathcal{R}_{a}$ at nozzle inlet calculated using ideal and noisy numerical signals. The case number corresponds to those investigated in Table 3. Error bars are obtained from 1000 runs with random noise. 


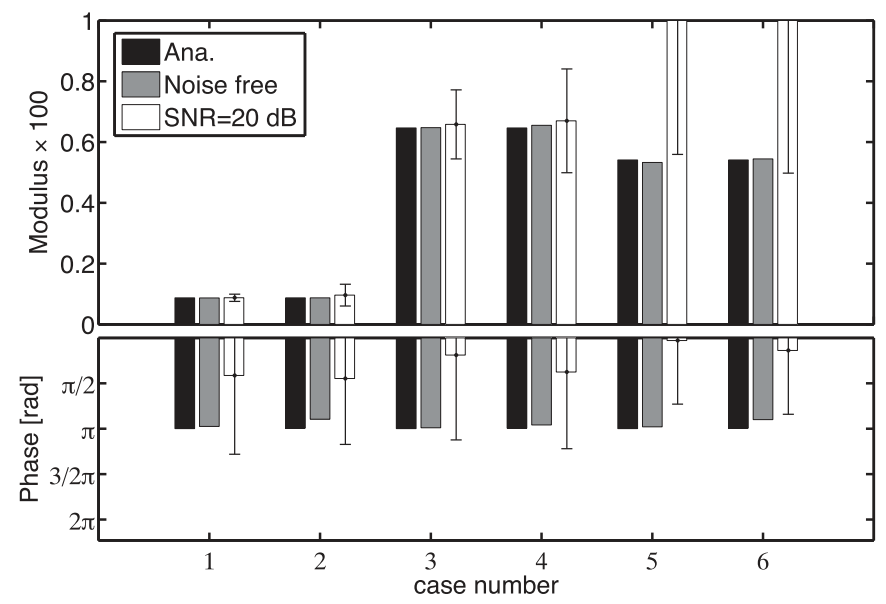

Fig. 14. Modulus (top) and phase (bottom) of the indirect reflection coefficient $\mathcal{R}_{S}$ calculated using ideal and noisy numerical signals. The case number corresponds to those investigated in Table 3. Error bars are obtained from 1000 runs with random noise.

temperature both oscillate within $10 \%$ about their mean values, the amplitude of the velocity fluctuations reaches more than $20 \%$ of the mean velocity. These velocity disturbances cannot be neglected compared to the mean velocity. As the entropy wave is transported at the convection velocity of the local flow, the phase difference between two measurement positions within the TAFG tube cannot be considered constant in time for large velocity modulations. To illustrate this phenomenon, both linear and nonlinear simulations of case 1 in Table 3 have been carried out with the SUNDAY code. From the primary variables (pressure, velocity and density), the characteristic waves $\Gamma^{ \pm}$are reconstructed with nonlinear and non-isentropic assumptions:

$$
2 \Gamma^{ \pm}=\frac{2}{\gamma-1}\left(\left(1+\frac{p^{\prime}}{p}\right)^{(\gamma-1) / 2 \gamma}-1\right) \pm \frac{u^{\prime}}{c e^{\sigma / 2}}
$$

They correspond to the characteristic waves of the hyperbolic system and reduce to $2 P^{ \pm}=p^{\prime} / p \pm u^{\prime} / c$ for small amplitude perturbations. The nonlinear expressions of the characteristic waves couple acoustic and entropy waves along their propagation route. The phase difference $\Delta \varphi_{12}$ between entropy fluctuations at positions $x_{1}$ and $x_{2}$ from linear and nonlinear simulations is plotted in Fig. 15 as a function of time. This phase difference calculated with the linear approximation is almost constant in time and has the same value $\Delta \varphi_{12}=-1.4 \mathrm{rad}$ as the theoretical phase difference $\Delta \varphi=-\omega\left(x_{2}-x_{1}\right) / u$ calculated with the bulk velocity $u=6.3 \mathrm{~m} / \mathrm{s}$ of the flow at the angular forcing frequency $\omega=2 \pi f$, where $f=20 \mathrm{~Hz}$. In the nonlinear simulations, this phase difference oscillates around the mean value $\Delta \varphi_{12}=-1.4$ rad. These results emphasize that at a given position, entropy fluctuations feature a phase lag that oscillates with time due to the large amplitude of velocity fluctuations.

One consequence of this velocity-entropy interaction is that the temperature signal deviates from an ideal harmonic wave as shown in Fig. 12. Another consequence is that the incoming pressure and entropy waves have a phase shift that varies in time at the entrance of the nozzle at $x_{n}$, although the amplitude of this time-varying phase difference remains small. This phenomenon has not been considered in the frequency domain analysis conducted to exploit the measurements with the diff-R method. This might lead to small errors in the determination of the indirect reflection coefficient $\mathcal{R}_{s}$. This error is difficult to assess because it depends on measurement positions. One way to reduce it is put the microphone and temperature probes as close as possible to the nozzle inlet. Reducing this distance mitigates to some extent the effect of wave steepening.

\section{Conclusion}

A new experimental setup generating simultaneously direct and indirect noises was presented. Temperature fluctuations are generated by a controllable harmonic acoustic modulation for forcing frequencies from 10 to $100 \mathrm{~Hz}$. The unsteady flow with temperature and pressure fluctuations is accelerated within a convergent nozzle that discharges freely to the atmosphere. Synchronized measurements of the pressure (microphones) and temperature (two-thermocouple sensor) fluctuations upstream of the nozzle allow determining the reflection coefficients of the nozzle as well as the acoustic and entropy waves approaching the nozzle.

A frequency-domain post-processing method called diff- $R$ was developed and used to separate direct from indirect noise contributions upstream of the nozzle. The method was developed by assuming small-amplitude linear perturbations. It was 


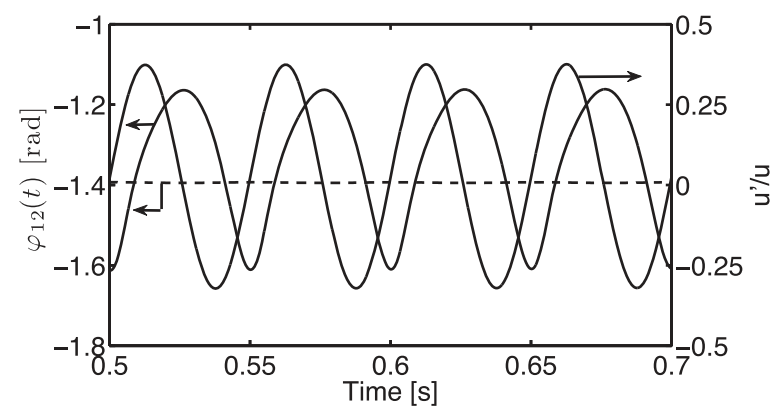

Fig. 15. Phase difference $\Delta \varphi_{12}$ between entropy waves at $x_{1}$ and $x_{2}$ (left axis) and velocity fluctuation $u^{\prime}$ at $x_{2}$ (right axis) as a function of time. Case NO.1 in Table 3. dashed line: linear simulation; solid line: nonlinear simulation.

validated by a set of direct numerical simulations of the nonlinear Euler equations with and without entropy fluctuations. These simulations also revealed the sensitivity of this method to signal noise.

The reflection coefficients measured at the inlet of the nozzle with and without entropy perturbations were found to be in good agreement with the analytical solutions for the acoustic reflection coefficient $\mathcal{R}_{a}$. Due to the limited level of the entropy noise contribution compared to the direct noise contribution in the test rig, the measurement of the entropy induced acoustic reflection coefficient $\mathcal{R}_{s}$ is largely affected by noise when the signal-to-noise ratio drops below $20 \mathrm{~dB}$. In the absence of noise, the diff-R method allows to well separate both the acoustic and entropy contributions, but theoretical predictions could not be recovered in the experiments due to the large SNR. The limitations of the actual test rig were identified. However the combined numerical and experimental investigations carried out in this work show that the diff-R processing method is a promising way for direct and indirect noise identification for synchronized entropy and acoustic perturbations.

\section{Appendix A. Quasi-steady analysis of TAFG setup}

It has been found in the parametric study of the TAFG setup that the amplitude of temperature fluctuations in the TAFG setup is very sensitive to changes of the mass flow rate of cross flow jets and mainstream. A quasi-steady analysis of the perturbed flow is used here as a support to interpret the observed features and optimize the system's performance in generating high amplitude temperature fluctuations.

A schematic view of the hot air injection zone is presented in Fig. A.16, in which the main stream is modeled by a constant mass flow rate $\dot{m}_{1}$, a constant temperature $T_{1}$ and an oscillating pressure $p_{1}(t)=p_{1}+p_{1}^{\prime}(t)$ due to loudspeaker forcing. In the low frequency limit $(\omega \rightarrow 0), p_{1}(t)$ is supposed to be uniform in the system but evolves with time. Crossflow jets at temperature $T_{2}$ are injected from the hot air plenum, and its pressure $p_{2}$ is supposed to remain unaffected by the pressure changes in the main stream. This is reasonable for injectors with small holes where the mean pressure drop $\Delta p=p_{2}-p_{1}$ is large compared to $p_{1}^{\prime}(t)$. Thus the total pressure drop from the plenum to the main stream $\Delta p-p_{1}^{\prime}(t)$ controls the momentum flux of the hot air injected through the jets, and the fluctuating mass flow rate of the jets can be calculated by:

$$
\dot{m}_{2}(t)=C_{D} A_{2} \sqrt{2 \rho_{2}\left(p_{2}-p^{\prime}(t)\right)}
$$

where $C_{D}$ and $A_{2}$ are the discharge coefficient and the total cross section surface area of the jet ducts, and $\rho_{2}$ is the density of the jet flow. Writing $\dot{m}_{2}=C_{D} A_{2} \sqrt{2 \rho_{2} \Delta p}$, one obtains for $\left|p_{1}^{\prime}(t)\right| / \Delta p \ll 1$ to the leading order:

$$
\dot{m}_{2}(t)=\dot{m}_{2}\left(1-\frac{1}{2} \frac{p_{1}^{\prime}(t)}{\Delta p}\right)
$$

Downstream of the crossflow jets, the mass flow rate $\dot{m}_{3}(t)$ and the gas temperature $T_{3}(t)$ of the air mixture are obtained by a mass and energy balance:

$$
\begin{aligned}
& \dot{m}_{3}(t)=\dot{m}_{1}+\dot{m}_{2}(t) \\
& C_{P} \dot{m}_{3}(t) T_{3}=C_{P} \dot{m}_{1} T_{1}+C_{P} \dot{m}_{2}(t) T_{2}
\end{aligned}
$$

where constant pressure heat capacities $C_{P}$ are supposed to be the same in the different flow regions under the explored conditions. Combining the balance equations gives the gas temperature $T_{3}(t)$ :

$$
T_{3}(t)=\frac{\dot{m}_{1} T_{1}+\dot{m}_{2}(t) T_{2}}{\dot{m}_{1}+\dot{m}_{2}(t)}
$$




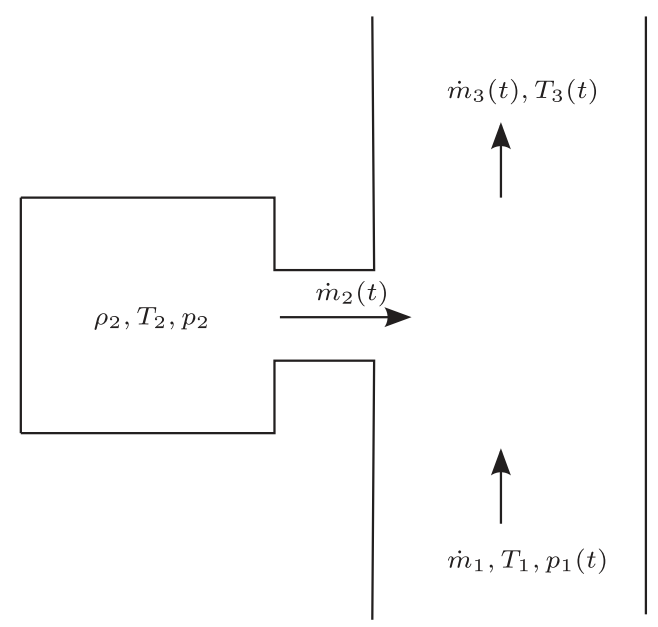

Fig. A.16. Schematic view of the crossflow jets injecti on to the main stream.

This expression is linearized for small pressure perturbations $p_{1}(t)$ by writing $T_{3}^{\prime}(t)=T_{3}(t)-T_{3}$ where $T_{3}=$ $\left(\dot{m}_{1} T_{1}+\dot{m}_{2} T_{2}\right) /\left(\dot{m}_{1}+\dot{m}_{2}\right)$ :

$$
\frac{T_{3}^{\prime}(t)}{T_{3}}=-\frac{1}{2} \frac{p^{\prime}(t)}{\Delta p}\left(\frac{\dot{m}_{2} T_{2}}{\dot{m}_{1} T_{1}+\dot{m}_{2} T_{2}}-\frac{\dot{m}_{2}}{\dot{m}_{1}+\dot{m}_{2}}\right)
$$

The factor $\alpha$ :

$$
\alpha=\frac{\dot{m}_{2} T_{2}}{\dot{m}_{1} T_{1}+\dot{m}_{2} T_{2}}-\frac{\dot{m}_{2}}{\dot{m}_{1}+\dot{m}_{2}}
$$

in the right-hand-side of Eq. (A.6) depends on the cross-flow to main stream mass flowrates and temperatures. When $T_{2} / T_{1}>1$ as in our experiments, $\alpha>0$.

Combining Eqs. (21) and (A.6) yields an expression for the transfer function $H_{s p}$ at the zero-frequency limit, which writes:

$$
H_{s p}=\frac{\hat{s} / c_{p}}{\hat{p} / \gamma p}=1-\gamma-\frac{\alpha}{2} \cdot \frac{\gamma p}{\Delta p}
$$

With the specific heat ratio for air $\gamma=1.4, H_{s p}$ is a negative real number, meaning that entropy and pressure waves are in phase opposition in the TAFG system in the zero-frequency limit. The modulus of $H_{s p}$ can be optimized by maximizing $\alpha$ :

$$
\alpha_{\max }=\frac{\sqrt{T_{2} / T_{1}}-1}{\sqrt{T_{2} / T_{1}}+1} \text { for } \dot{m}_{2} / \dot{m}_{1}=\sqrt{T_{1} / T_{2}}
$$

In the experiments $T_{1} \approx 290 \mathrm{~K}$ and $T_{2} \approx 390 \mathrm{~K}$, so the optimized injection condition is:

$$
\dot{m}_{2} / \dot{m}_{1}=0.86 \text { and } \alpha_{\max }=0.07
$$

\section{References}

[1] F. Marble, S. Candel, Acoustic disturbance from gas non-uniformities convected through a nozzle, J. Sound Vib. 55 (1977) 225-243, http://dx.doi.org/ 10.1016/0022-460X(77)90596-X.

[2] N. Cumpsty, Jet engine combustion noise: pressure, entropy and vorticity perturbations produced by unsteady combustion or heat addition, J. Sound Vib. 66 (1979) 527-544, http://dx.doi.org/10.1016/0022-460X(79)90697-7.

[3] Candel, Durox, Ducruix, Birbaud, Noiray, Schuller, Flame dynamics and combustion noise: progress and challenges, Int. J. Aeroacoustics 8 (2009) 1-56, http://dx.doi.org/10.1260/147547209786234984.

[4] A.P. Dowling, Y. Mahmoudi, Combustion noise, Proc. Combust. Inst. 35 (2015) 65-100, http://dx.doi.org/10.1016/j.proci.2014.08.016.

[5] W.C. Strahle, Combustion noise, Prog. Energy Combust. Sci. 4 (1978) 157-176, http://dx.doi.org/10.1016/0360-1285(78)90002-3.

[6] M. Leyko, F. Nicoud, T. Poinsot, Comparison of direct and indirect combustion noise mechanisms in a model combustor, AIAA J. 47 (2009) 2709-2716, http://dx.doi.org/10.2514/1.43729.

[7] M. Leyko, I. Durán, S. Moreau, F. Nicoud, T. Poinsot, Simulation and modelling of the waves transmission and generation in a stator blade row in a combustion-noise framework, J. Sound Vib. 333 (2014) 6090-6106, http://dx.doi.org/10.1016/j.jsv.2014.06.034.

[8] E.E. Zukoski, J.M. Auerbach, Experiments concerning the response of supersonic nozzles to fluctuating inlet conditions, J. Eng. Power (1976) 60-64.

[9] F. Bake, C. Richter, B. Mühlbauer, N. Kings, I. Röhle, F. Thiele, B. Noll, The Entropy Wave Generator (EWG): a reference case on entropy noise, J. Sound Vib. 326 (2009) 574-598, http://dx.doi.org/10.1016/j.jsv.2009.05.018. 
[10] M. Leyko, F. Nicoud, S. Moreau, T. Poinsot, Numerical and analytical investigation of the indirect combustion noise in a nozzle, Comptes Rendus Mécanique 337 (2009) 415-425, http://dx.doi.org/10.1016/j.crme.2009.06.025.

[11] I. Durán, S. Moreau, T. Poinsot, Analytical and numerical study of combustion noise through a subsonic nozzle, AIAA J. 51 (2013) 42-52, http://dx.doi. org/10.2514/1.J051528.

[12] A. Giauque, M. Huet, F. Clero, Analytical analysis of indirect combustion noise in subcritical nozzles, J. Eng. Gas Turbines Power 134 (2012) 111202, http: //dx.doi.org/10.1115/1.4007318.

[13] M. Muthukrishnan, W.C. Strahle, D.H. Neale, Separation of hydrodynamic, entropy, and combustion noise in a gas turbine combustor, AIAA J. 16 (1978) 320-327, http://dx.doi.org/10.2514/3.60895.

[14] P.A. Hield, M.J. Brear, Comparison of open and choked premixed combustor exits during thermoacoustic limit cycle, AIAA J. 46 (2008) 517-526, http: //dx.doi.org/10.2514/1.32650.

[15] M. Zhu, A. Dowling, K.N.C. Bray, Self-excited oscillations in combustors with spray atomizers, J. Eng. Gas Turbines Power 123 (2001) 779, http://dx.doi. org/10.1115/1.1376717.

[16] J. Eckstein, T. Sattelmayer, Combustion instabilities in aeroengines, J. Propuls. Power 22 (2006) 2006.

[17] H.S. Tsien, The transfer functions of rocket nozzles, J. Am. Rocket Soc. (1952) 139-143, http://dx.doi.org/10.2514/8.4448.

[18] L. Crocco S.-I. Cheng Theory of Combustion Instability in Liquid Propellant Rocket Motors 21, 1956, Butterworths Scientific Publications, London; Interscience Publishers Inc New York.

[19] W. Bell, B. Daniel, B. Zinn, Experimental and theoretical determination of the admittances of a family of nozzles subjected to axial instabilities, J. Sound Vib. 30 (1973) 179-190.

[20] W.H. Moase, M.J. Brear, C. Manzie, The forced response of choked nozzles and supersonic diffusers, J. Fluid Mech. 585 (2007) 281, http://dx.doi.org/ $10.1017 /$ S0022112007006647.

[21] S.R. Stow, A.P. Dowling, T.P. Hynes, Reflection of circumferential modes in a choked nozzle, J. Fluid Mech. 467 (2002) 215-239, http://dx.doi.org/ $10.1017 /$ S0022112002001428.

[22] C.S. Goh, A.S. Morgans, Phase prediction of the response of choked nozzles to entropy and acoustic disturbances, J. Sound Vib. 330 (2011) 5184-5198, http://dx.doi.org/10.1016/j.jsv.2011.05.016.

[23] M. Huet, A. Giauque, A nonlinear model for indirect combustion noise through a compact nozzle, J. Fluid Mech. 733 (2013) 268-301, http://dx.doi.org/ $10.1017 / \mathrm{jfm} .2013 .442$.

[24] A.P. Dowling, The calculation of thermoacoustic oscillations, J. Sound Vib. 180 (1995) 557-581, http://dx.doi.org/10.1006/jsvi.1995.0100.

[25] C.S. Goh, A.S. Morgans, The influence of entropy waves on the thermoacoustic stability of a model combustor, Combust. Sci. Technol. 185 (2013) 249-268, http://dx.doi.org/10.1080/00102202.2012.715828.

[26] E. Motheau, F. Nicoud, T. Poinsot, Mixed acoustic-entropy combustion instabilities in gas turbines, J. Fluid Mech. 749 (2014) 542-576, http://dx.doi.org/ 10.1017/jfm.2014.245.

[27] P. Dowling, R. Stow, Acoustic analysis of gas turbine combustors, J. Propuls. Power 19 (2003) $751-764$.

[28] B.-T. Chu, L.S.G. Kovásznay, Non-linear interactions in a viscous heat-conducting compressible gas, J. Fluid Mech. 3 (1958) 494, http://dx.doi.org/ $10.1017 /$ S0022112058000148.

[29] H. Levine, J. Schwinger, On the radiation of sound from an unflanged circular pipe, Phys. Rev. 73 (1948) 383-406, http://dx.doi.org/10.1103/ PhysRev.73.383.

[30] M.C.A.M. Peters, A. Hirschberg, A.J. Reijnen, A.P.J. Wijnands, Damping and reflection coefficient measurements for an open pipe at low Mach and low Helmholtz numbers, J. Fluid Mech. 256 (1993) 499, http://dx.doi.org/10.1017/S0022112093002861.

[31] E. Motheau, F. Nicoud, T. Poinsot, Using boundary conditions to account for mean flow effects in a zero mach number acoustic solver, J. Eng. Gas Turbines Power 134 (2012) 111502, http://dx.doi.org/10.1115/1.4007198.

[32] Z. Yao, Y. Gao, M. Zhu, A.P. Dowling, K.N.C. Bray, Combustion rumble prediction with integrated computational-fluid-dynamics/low-order-model methods, J. Propuls. Power 28 (2012) 1015-1025, http://dx.doi.org/10.2514/1.B34469.

[33] J.Y. Chung, D.a. Blaser, Transfer function method of measuring in-duct acoustic properties. I. Theory, J. Acoust. Soc. Am. 68 (1980) 907-913, http://dx. doi.org/10.1121/1.384778.

[34] D.J. Bodony, Scattering of an entropy disturbance into sound by a symmetric thin body, Phys. Fluids 21 (2009) 1-10, http://dx.doi.org/10.1063/ 1.3225143.

[35] A. Scarpato, N. Tran, S. Ducruix, T. Schuller, Modeling the damping properties of perforated screens traversed by a bias flow and backed by a cavity at low Strouhal number, J. Sound Vib. 331 (2012) 276-290, http://dx.doi.org/10.1016/j.jsv.2011.09.005.

[36] L.J. Forney, G.C. Fralick, Multiwire thermocouples in reversing flow, Rev. Sci. Instrum. 66 (1995) 5050, http://dx.doi.org/10.1063/1.1146129.

[37] M. Tagawa, T. Shimoji, Y. Ohta, A two-thermocouple probe technique for estimating thermocouple time constants in flows with combustion: in situ parameter identification of a first-order lag system, Rev. Sci. Instrum. 69 (1998) 3370, http://dx.doi.org/10.1063/1.1149103.

[38] P.G. O'Reilly, R.J. Kee, R. Fleck, P.T. McEntee, Two-wire thermocouples: a nonlinear state estimation approach to temperature reconstruction, Rev. Sci. Instrum. 72 (2001) 3449, http://dx.doi.org/10.1063/1.1384428.

[39] P.C. Hung, G. Irwin, R. Kee, S. McLoone, Difference equation approach to two-thermocouple sensor characterization in constant velocity flow environments, Rev. Sci. Instrum. 76 (2005) 1-10, http://dx.doi.org/10.1063/1.1847412.

[40] W. Tao, Time Resolved Temperature and Pressure Based Methodology for Direct and Indirect Combustion Noise Separation (Ph.D. thesis), Université Paris-Saclay, 2016. 